\title{
Noncommutative graded domains with quadratic growth
}

\author{
M. Artin', J.T. Stafford ${ }^{2}$ \\ 'Department of Mathematics, MIT, Cambridge, MA 02139, USA \\ ${ }^{2}$ Department of Mathematies, University of Michigan, Ann Arbor, MI 48109, USA
}

Dedicated to the memory of Shimshon Amitsur

Oblatum 5-XI-1994 \& 28-111-1995

\section{Contents}

0. Introduction 231

1. The graded quotient ring of a graded prime ring of dimension two 235

2. The associated divisors $\quad 244$

3. The stable image of the curve 254

4. Domains of dimension two which are generated in degree one 257

5. The case of an automorphism of infinite order 264

6. Applications to the structure of graded domains of dimension two 270

Abstract. Let $k$ be an algebraically closed field, and let $R$ be a finitely generated, connected graded $k$-algebra, which is a domain of Gelfand-Kirillov dimension two. Write the graded quotient ring $Q(R)$ of $R$ as $D\left[z, z^{-1} ; \sigma\right]$, for some automorphism $\sigma$ of the division ring $D$. We prove that $D$ is a finitely generated field extension of $k$ of transcendence degree one. Moreover, we describe $R$ in terms of geometric data. If $R$ is generated in degree one then, up to a finite dimensional vector space, $R$ is isomorphic to the twisted homogeneous coordinate ring of an invertible sheaf $\mathscr{L}$ over a projective curve $Y$. This implies, in particular, that $R$ is Noetherian, that $R$ is primitive when $|\sigma|=\infty$ and that $R$ is a finite module over its centre when $|\sigma|<\infty$. If $R$ is not generated in degree one, then $R$ will still be Noetherian and primitive if $\sigma$ has infinite order, but $R$ need not be Noetherian when $\sigma$ has finite order.

\section{Introduction}

It is intuitively clear that the ring of functions on an affine algebraic curve can have no noncommutative analogue, because the functions on a curve depend on one variable, and functions of one variable commute with each other. Indeed, a 
fundamental theorem of Small and Warfield [SW] asserts that a finitely generated prime algebra $S$ over a field $k$ which has linear growth is a finite module over its centre, and that its centre is a finitely generated $k$-algebra of dimension one. Moreover, if the ground field is algebraically closed and if $S$ is a domain, then $S$ is commutative. Thus their result provides a precise formulation of the intuitive picture for affine curves. It is extended by the theorem of Bergman $[B e],[K L]$, according to which the Gelfand-Kirillov dimension, which measures the growth of an algebra, cannot lie in the range $1<d<2$. But a projective curve is defined by a graded ring of dimension 2 , and noncommutative graded algebras with quadratic growth do exist. The quantum polynomial rings, such as

$$
A=k\langle x, y\rangle /\left(y x-x y-x^{2}\right),
$$

are well-known examples.

We will call a graded $k$-algebra $R=\bigoplus_{n \geq 0} R_{n}$ finitely graded if it is a finitely generated algebra, and if $R_{0}$ is a finite dimensional vector space over $k$. The purpose of this paper is to describe all Noetherian finitely graded domains of GK-dimension 2 over an algebraically closed field, together with their projective geometry, thereby giving a projective analogue of the theorem of Small and Warfield. We also describe the geometric conditions which a finitely graded domain of GK-dimension 2 must satisfy in order to be Noetherian.

As in commutative algebra, a natural first step towards describing a graded domain $R$ is to determine its "birational" structure, that is, to determine the graded ring of fractions $Q$, the ring obtained by inverting its homogeneous elements. The graded ring of fractions of a graded Goldie domain is described in $[\mathrm{NV}]$ as a skew Laurent polynomial ring $D\left[z, z^{-1} ; \sigma\right]$, in which $\sigma$ is an automorphism of a division ring $D$, and multiplication is defined by $z d=d^{\sigma} z$. If $R$ were commutative and two-dimensional, then $D$ would be the function field of the associated projective curve, and $\sigma$ would be the identity automorphism. The first result of the paper generalizes this description to noncommutative rings:

Theorem 0.1. Let $R$ be a finitely graded domain such that $2 \leqq G K \operatorname{dim}(R)<$ $\frac{11}{5}$, with graded quotient ring $D\left[z, z^{-1} ; \sigma\right]$.

(i) The division ring $D$ is a finitely generated module over its centre $K$, and $K$ is a finitely generated field extension of $k$ of transcendence degree one. If $k$ is algebraically closed, then $D=K$.

(ii) Conversely, if $D$ is as in (i) and if $S$ is any finitely graded subring of $D\left[z, z^{-1} ; \sigma\right]$, then $G K \operatorname{dim}(S) \leqq 2$. More precisely, there exists a constant $c$ such that $\operatorname{dim}_{k} S_{n} \leqq$ cn, for all $n \geqq 1$.

The theorem can be regarded as saying that, when $k$ is algebraically closed, $R$ is determined birationally by an automorphism of an algebraic curve. The natural generalization of the theorem to semi-prime Goldie rings also holds (see Theorem 1.1 and Remark 1.17 for the precise statement).

As is illustrated by examples in Sect. 1, Theorem 0.1 does not extend directly to domains of GK-dimension $\geqq 3$. However, the number $\frac{11}{5}$ seems to 
appear because of technical limitations in our proof. Since this number has no obvious intrinsic significance, we are led to conjecture that a graded domain cannot have GK-dimension strictly between 2 and 3 .

In outline, the proof of Theorem 0.1 runs as follows: Let $b$ be a nonzero homogeneous element of $R$. Since $R$ is a graded Ore domain, we may find a common right denominator for the fractions $b^{-1} R_{n}$. In other words, there exists an integer $t_{n}$ so that $b^{-1} R_{n} \subseteq R_{t_{n}} \beta_{n}^{-1}$ for some homogeneous element $\beta_{n}$ of the appropriate degree. The main step in the proof is a careful estimate of the growth of the function $t_{n}$ (see Proposition 1.4). Using this estimate, we show that the division ring $D$ has GK-dimension $<2$. This being the case, Bergman's theorem shows that $G K \operatorname{dim}(D) \leqq 1$, which puts one in position to apply the theorem of Small and Warfield to complete the proof.

We now pass to the geometric description of a ring $R$ satisfying the hypotheses of Theorem 0.1. For this, we need to assume that $D=K$ in Theorem 0.1 and so, for the rest of the introduction, we will assume that $k$ is algebraically closed. Thus, $D$ is now the function field of a smooth, projective curve $X$. The model for our program is again provided by algebraic geometry. The classic results of Serre [Se] describe the commutative situation in the following way: Let $R$ be a commutative graded domain of dimension 2, which is generated by finitely many elements of degree 1 . There exist a projective curve $Y$ and an invertible sheaf $\mathscr{L}$ on $Y$ such that, for large $n, R_{n} \cong H^{0}\left(Y, \mathscr{L}^{\otimes n}\right)$. The next result (Theorem 4.7) extends Serre's theorem to noncommutative rings, using Van den Bergh's notion [AV] of a twisted homogeneous coordinate ring:

Theorem 0.2. Let $R$ be a finitely graded domain of $G K$-dimension 2, which is finitely generated by elements of degree 1. There exist a projective curve $Y$, an automorphism $\sigma$ of $Y$, and an invertible sheaf $\mathscr{L}$ on $Y$, such that for $n \gg 0, R_{n} \cong H^{0}\left(Y, \mathscr{L} \otimes \mathscr{L}^{\sigma} \otimes \cdots \otimes \mathscr{L}^{\sigma^{n-1}}\right)$.

Thus $R$ has finite codimension in the twisted homogeneous coordinate ring $B(Y, \mathscr{L}, \sigma)$; the graded ring defined by $B_{n}=H^{0}\left(Y, \mathscr{L} \otimes \mathscr{L}^{\sigma} \otimes \cdots \otimes \mathscr{L}^{\sigma^{n-1}}\right)$. Its structure is well understood, and as a consequence, one obtains:

Corollary 0.3. [AV], [ST] Let $R$ be as in Theorem 0.2. Then $R$ is Noetherian, and the quotient category $\overline{\mathrm{gr}}-R$ of finitely generated graded right $R$-modules modulo those of finite length is equivalent to the category mod- $\mathcal{O}_{Y}$ of coherent sheaves on $Y$. Moreover, if $|\sigma|<\infty$ then $R$ is finite module over its centre.

The case that $R$ is not generated in degree one, however, differs essentially from commutative algebra, and exhibits a striking new phenomenon. Of course it may happen, as it always the case if $R$ is commutative, that some Veronese ring $R^{(d)}=\bigoplus_{n \geqq 0} R_{n d}$ is generated in degree one; that is, generated by $R_{1}^{(d)}=$ $R_{d}$. If so, then $R$ is a finite $R^{(d)}$-module and, as in the commutative case, the structure of $R$ is closely related to that of $R^{(d)}$. So Theorem 0.2 can be applied to such rings. (See Section 6 for further details.)

Unlike the commutative case, though, it may also happen that no Veronese ring is generated in degree one; indeed, this occurs frequently. A typical 
example is the subring $R=k+y A$ of the quantum polynomial ring $A$ which was mentioned above. This ring was studied in [SZ], where it is shown that $R$ is Noetherian if and only if the characteristic of the field $k$ is zero. Thus, though this is a graded domain of GK-dimension 2, which has a finite presentation with integer coefficients, and whose presentation and Hilbert function are independent of the characteristic, the Noetherian property fails to be preserved under reduction modulo $p$ for any prime $p$ ! Our results show that this dichotomy occurs generally, although it is actually controlled by the order of $\sigma$ rather than the characteristic of the field.

The next two theorems summarize the main results of Sects 5 and 6 .

Theorem 0.4. Let $R$ be a finitely graded domain of GK-dimension 2 .

(i) If $\sigma$ has infinite order, then $R$ is a Noetherian, primitive ring, and every Veronese ring of $R$ is finitely generated.

(ii) If $\sigma$ has finite order $d$ and if no Veronese ring of $R$ is generated in degree one, then $R$ is not Noetherian. Indeed, $R^{(d)}$ is a commutative ring which is not finitely generated.

The structure of $R$ when $|\sigma|=\infty$ is made considerably more precise in the paper, but for simplicity we only state the implications for a Veronese ring below. A complete description of the rings which arise in case (ii) of Theorem 0.4 remains to be found.

Theorem 0.5. Let $R$ be as in Theorem 0.4. Assume that no Veronese ring of $R$ is generated in degree one, and that $|\sigma|=\infty$. Then, after replacing $R$ by an appropriate Veronese ring, the following hold:

(i) There is an algebraic curve $Y$ and there is a sequence of invertible sheaves $\left\{\mathscr{L}_{m}\right\}$ on $Y$, such that $R_{m} \cong H^{0}\left(Y, \mathscr{L}_{m}\right)$, for all $m \geqq 1$.

(ii) $R$ is generated in degrees one and two.

(iii) $R$ is contained in a twisted homogeneous coordinate ring $B=B(Y, \mathcal{A}$, $\sigma$ ) in such a way that $B$ is finitely generated as a right $R$-module but infinitely generated as a left R-module.

(iv) $\overline{\mathrm{gr}}-R$ is equivalent to mod-(i) ${ }_{\gamma}$.

The idea behind the proof of the last three theorems is as follows. For simplicity, let us suppose that $R_{1} \neq 0$ (see Proposition 2.21 and Section 6 for the methods by which one can reduce to this case). Fix a non-zero $z \in R_{1}$ and write $R_{m}=\bar{R}_{m} z^{m}$, so that $\bar{R}_{m}$ is a subspace of the function field $D=k(X)$. The subspace $\bar{R}_{n}$ generates a fractional ideal $\mathfrak{O}_{X} \bar{R}_{n}$ in the structure sheaf $\mathcal{O}_{X}$, and therefore determines a divisor $D_{n}$. Much of the structure of the ring $R$ is encoded in these divisors and a detailed analysis of the asymptotic structure of $\left\{D_{n}\right\}$ is given in Sect. 2. For example, while Theorem 0.4(ii) follows easily from the fact that the Veronese ring $R^{(d)}$ is commutative, the explanation for this phenomenon really stems from peculiarities inherent in $\left\{D_{n}\right\}$ (see Propositions 2.6 and 2.8).

For the rest of the introduction, assume either that $R$ is generated in degree one or that $\sigma$ has infinite order. A key observation in the latter case, and the ultimate reason why Theorem 0.4 (i) is true, is that the finite orbits of $\sigma$ 
are actually fixed points. This is significant because, at these fixed points, the divisors $D_{n}$ behave as they would if $\sigma$ were the identity, i.e, as if $R$ were commutative, and so peculiarities like those of Theorem 0.4(ii) cannot occur. On the other hand, the structure of the $D_{n}$ on an infinite orbit of $\sigma$ is tightly constrained (see Proposition 2.11).

The space $\bar{R}_{m}$ also defines a morphism $\pi_{m}: X \rightarrow Y_{m} \subset \mathbb{P}\left(\bar{R}_{m}^{*}\right)$ and the information about divisors from Section 2 is used in Section 3 to show that the $Y_{m}$ are isomorphic for large $m$. (If $R$ is not generated in degree one then, as with commutative rings, one may have to replace $R$ by some Veronese ring before this holds.) This defines the curve $Y$ of Theorems 0.2 and 0.5 ; simply take $Y=Y_{m}$ for some $m \gg 0$. Moreover, one now has induced morphisms $\phi_{m}: Y \rightarrow \mathbb{P}\left(\bar{R}_{m}^{*}\right)$ and it follows that the fractional ideal $\mathscr{L}_{m}=\mathfrak{C}_{Y} \bar{R}_{m}$ is the pull back to $Y$ of the Serre twisting sheaf on $\mathbb{P}\left(\bar{R}_{m}^{*}\right)$. In particular, $\mathscr{L}_{m}$ is invertible and is the sheaf $\mathscr{L}_{m}$ of Theorem 0.5. A major step in the proofs of Theorems $0.2-0.5$ is to show that $\bar{R}_{m}=H^{0}\left(Y, \mathscr{L}_{m}\right)$ (see Theorem 4.1 and Proposition 5.4).

When $R$ is generated in degree one, $\bar{R}_{m}=\bar{R}_{1} \bar{R}_{1}^{\sigma} \cdots \bar{R}_{1}^{\sigma^{m-1}}$ and so $\mathscr{L}_{m}=$ $\mathscr{L}_{1} \otimes \cdots \otimes \mathscr{L}_{1}^{\sigma^{m-1}}$. Thus, Theorem 0.2 holds with $\mathscr{L}=\mathscr{L}_{1}$. In the context of Theorem 0.5 , define $\mathscr{A}_{r}$ by $\mathscr{N}_{r}^{r}=\mathscr{L}_{r+1} \mathscr{L}_{r}^{-1}$. Then one can show that $A_{r}=A_{r+1}=\mathcal{A}$, for any large $r$. However, the fact that no Veronese ring of $R$ is generated in degree one implies that $\mathscr{L} \supsetneq \mathscr{L}_{1}$. This is more or less equivalent to part (iii) of Theorem 0.5 .

\section{The graded quotient ring of a graded prime ring of dimension two}

In this section, we describe the graded quotient ring of a finitely graded, prime Goldie ring of Gelfand-Kirillov dimension 2. This proves, as a special case. Theorem 0.1 of the introduction. We begin with some comments about quotient rings of graded rings.

Let $A$ be a $\mathbb{Z}$-graded ring with at least one homogeneous, regular element in positive degree. Then, $A$ is a graded division ring if every homogeneous element is invertible. By [NV, A.I.4.3], such a graded division ring $A$ has the form of a skew Laurent polynomial ring $A=D\left[z, z^{-1} ; \sigma\right]$, where $\sigma$ is an automorphism of a division ring $D$ and $z$ is an element of positive degree. By [NV, A.I.5.8], $A$ is a graded simple Artinian ring if $A \cong M_{n}(\Delta)$, the $m \times m$ matrix algebra over a graded division ring $\Delta$, except that the degrees are shifted by some vector $\left(d_{1}, \ldots, d_{m}\right) \in \mathbb{Z}^{m}$. This means that a matrix $\left(a_{l j}\right) \in A$ is a homogeneous element of degree $n$ if $a_{i j} \in \Delta_{n+d_{t}-d_{1}}$. Now let $R$ be a graded, prime ring. By [LVV, II.1.2], $R$ is Goldie if and only if it is graded Goldie. If $R$ is Goldie, with at least one homogeneous regular element of strictly positive degree, then [NV, C.I.1.6] implies that the homogeneous regular elements $\mathscr{C}$ of $R$ form an Ore set and that the graded quotient ring $Q(R)=R^{\mathscr{C}} \mathscr{G}^{-1}$ of $R$ is a graded simple Artinian ring. 
Throughout this section, $D$ will denote a division ring with an automorphism $\sigma$, and $\Delta$ will denote the $\mathbb{Z}$-graded division ring $D\left[z, z^{-1} ; \sigma\right]$. A finitely generated field extension of $k$ of transcendence degree 1 will be called a function field in one variable. Throughout, dim denotes dimension as a $k$-vector space, while $Z(A)$ will denote the centre of a ring $A$.

The object of this section is to prove the following theorem:

Theorem 1.1. Let $R$ be a finitely graded, prime Goldie ring with $1<$ $G K \operatorname{dim}(R)<\frac{11}{5}$, that contains a homogeneous, regular element of positive degree. Write $Q(R)=M_{n}(\Delta)$, the shifted matrix ring over a graded division ring $\Delta=D\left[z, z^{-1} ; \sigma\right]$. Then the division ring $D$ is finite over its centre $Z(D)=K$, and $K$ is a function field in one variable. If $k$ is algebraically closed, then $D=K$.

Note that a domain $R$ of finite Gelfand-Kirillov dimension is automatically an Ore domain, [KL, 4.12], and so Theorem 1.1 incorporates Theorem 0.1(i). However, there do exist prime, finitely graded rings of Gelfand-Kirillov dimension two that are not Goldie (see Example 1.18) and so the Goldie condition is necessary in the prime case. The proof in the case that $R$ is a domain will take up most of the section, since standard techniques can then be used to extend the result to the prime case.

We will also prove the following converse to Theorem 1.1, which completes the proof of Theorem 0.1 :

Theorem 1.2. Let $A=M_{n}\left(D\left[z, z^{-1} ; \sigma\right]\right)$ be a graded, simple Artinian ring, where $D$ is a finite module over its centre $K$ and $K$ is a function field in one variable. Then, any finitely graded subring $R$ of $A$ has quadratic growth, in the sense that there is a constant $c$ such that $\operatorname{dim}\left(R_{n}\right) \leqq c n$ for all $n>0$. In particular, GK $\operatorname{dim}(R) \leqq 2$.

A few comments are in order before we begin the proofs. First, Theorem 1.1 exhibits a dichotomy depending on the order of $\sigma$. For simplicity, we only state this for algebraically closed fields.

Corollary 1.3. Keep the hypotheses of Theorem 1.1 and assume that $D=K$. Then $R$ satisfies a polynomial identity if $|\sigma|<\infty$, but $Z(R)=k$ if $|\sigma|=\infty$.

Proof. The Corollary follows from the observation that $K[z ; \sigma]$ is a finite module over its centre if $|\sigma|<\infty$, while $K[z ; \sigma]^{\sigma}=K^{\sigma}=k$ if $|\sigma|=\infty$.

By Bergman's Theorem [KL, 2.5; Be], no ring can have Gelfand-Kirillov dimension strictly between one and two, and so, in fact, $2 \leqq G K \operatorname{dim}(R)<\frac{11}{5}$ in Theorem 1.1. This also implies that, in Theorem 1.2, GK $\operatorname{dim}(R)$ must be 0,1 , or 2 , and of course these cases are easily distinguished. The number $\frac{11}{5}$ in Theorem 1.1 has no obvious significance and we conjecture that, if $R$ is a finitely graded domain with $G K \operatorname{dim}(R)<3$, then $G K \operatorname{dim}(R) \leqq 2$. Indeed, we know of no domain $S$ whose Gelfand-Kirillov dimension is not an integer. However, as is shown by example at the end of the section, this is about the only improvement that could be made to these results. 
We now turn to the proof of Theorem 1.1. The main point is to show that, if $R$ is a domain satisfying the hypotheses of $(1.1)$, then $G K \operatorname{dim}(D)=1$. This will be reduced to a certain combinatorial problem, and the following result provides the intermediate step:

Proposition 1.4. Let $S$ be a finitely graded domain which is generated by $S_{1}$ as a k-algebra, and with $2 \leqq G K \operatorname{dim}(S)<\frac{11}{5}$. Fix a nonzero element $b \in S_{1}$. There exists a constant $d>0$ and a sequence of integers $\left\{\tau_{n}\right\}$ with the following properties:

(i) $\tau_{1}=1$ and $\tau_{n} \leqq d n^{5 / 3}$ for all $n \geqq 1$;

(ii) For all $n \geqq 1$, there exist non-zero homogeneous elements $\beta_{n} \in$ $S_{\tau_{n+1}-\tau_{n}}$, such that $S_{\tau_{n}} \beta_{n} \subseteq b S_{i^{\prime} n}$, where $v_{n}=\tau_{n+1}-1$.

We remark that, for any graded Ore domain $S$, part (ii) of Proposition 1.4 will hold for some sequence $\left\{\tau_{n}\right\}$ (write a basis of $b^{-1} S_{\tau_{n}}$ over a common right denominator) and so the estimate of part (i) is the key point. The required upper bound on $G K \operatorname{dim}(D)$ follows easily from this result:

Corollary 1.5. Let $R$ be a finitely graded domain with $1<G K \operatorname{dim}(R)<\frac{11}{5}$ and graded quotient ring $Q(R)=D\left[z, z^{-1} ; \sigma\right]$. Then, $G K \operatorname{dim}(D) \leqq 1$.

Proof. We prove the corollary, assuming that Proposition 1.4 is true. It suffices to prove that, if $T$ is a finitely generated subalgebra of $D$, then $G K \operatorname{dim}(T) \leqq 1$. Let $T$ be generated by a finite dimensional $k$-subspace $U$. We may assume that $1 \in U$, in which case we filter $T$ by $A_{i}=U^{i}$. Since $R$ is a graded Ore domain, we may write the elements of $U$ over a common denominator, say $U=A b^{-1}$, where $b \in R_{d}$ and $A \subseteq R_{d}$, for some $d>0$. Let $S$ be the subring of $R$ generated by $R_{d}$. Clearly, $S$ is a graded subring of $R$ and so $G K \operatorname{dim}(S)<\frac{11}{5}$. If $G K \operatorname{dim}(S)<2$ then, by Bergman's Theorem, $G K \operatorname{dim}(S) \leqq 1$. Thus, $S$ is a finite module over its centre, by [SW]. Therefore, if $E$ denotes the full quotient ring of $S$, then $G K \operatorname{dim}(T) \leqq G K \operatorname{dim}(E) \leqq 1$, by [KL, Proposition 4.2].

Thus, we may assume that $G K \operatorname{dim}(S) \geqq 2$. We regrade $S$ by defining $S=\bigoplus S_{i}$ for $S_{t}=\left(R_{d}\right)^{t}$. The hypotheses of Proposition 1.4 are now satisfied and we choose the $\tau_{n}$ and $\beta_{n}$ as defined there. Thus, $b^{-1} S_{\tau_{n}} \subset S_{\tau_{n+1}-1} \beta_{n}^{-1}$, for each $n$. By induction,

$$
A_{n+1} \subseteq\left(S_{1} b^{-1}\right)\left(S_{1} b^{-1}\right)^{n} \subseteq\left(S_{1} b^{-1}\right)\left(S_{\tau_{n}} \gamma_{n}^{-1}\right) \subseteq S_{1} S_{t_{n+1}-1} \beta_{n}^{-1} \gamma_{n}^{-1}=S_{t_{n+1}} \gamma_{n+1}^{-1}
$$

where $\gamma_{1}=b$ and $\gamma_{n+1}=\gamma_{n} \beta_{n}$ for $n>1$. Thus, $\operatorname{dim} A_{m} \leqq \operatorname{dim} R_{\tau_{m}}$, for all $m \geqq 1$.

Finally, pick $\varepsilon>0$ such that $G K \operatorname{dim}(R)<\frac{11}{5}-\varepsilon$. By the next lemma, there exists a constant $c>0$ such that $\operatorname{dim} R_{n} \leqq c n^{6 / 5-1}$, for all $n$. Thus, by Proposition 1.4(i), $\operatorname{dim} \Lambda_{m} \leqq \operatorname{dim} R_{\tau_{m}} \leqq c\left(d m^{5 / 3}\right)^{6 / 5-6} \leqq c^{\prime} m^{2-\varepsilon}$, for all $m \geqq 0$ and $c^{\prime}=c d^{6 / 5}$. In other words, $G K \operatorname{dim}(T)<2$ and so, by Bergman's Theorem, $G K \operatorname{dim}(T) \leqq 1$, as required. 
Lemma 1.6. Let $R$ be a finitely graded ring which contains a homogeneous regular element of positive degree. Let $d=G K \operatorname{dim}(R)$, and let e denote the greatest lower bound of the real numbers $r$ such that for some $c>0$, $\operatorname{dim}\left(R_{n}\right)<c n^{r}$ for all $n$. Then $e=d-1$.

We remark that the conclusion of the lemma can fail if $R$ contains no regular homogeneous element.

Proof. Let $a_{n}=\operatorname{dim}\left(R_{n}\right)$ and $A_{n}=a_{0}+\cdots+a_{n}$. By definition, $G K \operatorname{dim}(R)$ is the greatest lower bound of the real numbers $s$ such that, for some $c, A_{n} \leqq c n^{s}$ for all $n$. Now, if $a_{n} \leqq c n^{r}$, then

$$
A_{n}=\sum_{j=0}^{n} a_{j} \leqq \sum_{j=0}^{n} c j^{r} \leqq c^{\prime} n^{r+1} .
$$

Hence $d \leqq e+1$. For the opposite inequality, suppose that $A_{n} \leqq c n^{s}$ for all $n$. Let $b$ be a homogeneous regular element of degree $j>0$ in $R$. Since multiplication by $b$ is injective, $a_{n} \leqq a_{n+j}$ for all $n$. Then

$$
n a_{n} \leqq a_{n+j}+a_{n+2 j}+\cdots+a_{n+n j} \leqq A_{n(j+1)} \leqq c(j+1)^{s} n^{s}=c^{\prime} n^{s} .
$$

Hence $a_{n} \leqq c^{t} n^{s-1}$, which shows that $e \leqq d-1$.

We now turn to the proof of Proposition 1.4. Keep the notation of that proposition and let $V$ denote the graded right $S$-module $S / b S$. For $n \geqq 0$, define

$$
F(n)=\operatorname{dim}\left(S_{n}\right) \quad \text { and } \quad f(n)=\operatorname{dim}\left(V_{n}\right) ;
$$

thus $f(n)=F(n)-F(n-1)$ and $F(n)=f(0)+\cdots+f(n)$.

Lemma 1.7. $f(n) \geqq 1$ and $F(n) \geqq n+1$ for all $n \geqq 0$.

Proof. The lemma amounts to the assertion that $S_{n} \neq b S_{n-1}$ for every $n \geqq 0$. If $S_{n}=b S_{n-1}$ for some $n$, then, because $S$ is generated in degree 1, $S_{n+k}=$ $b S_{n+k-1}$ for every $k \geqq 0$. Hence, $F(m)$ is constant for $m \geqq n$ and, by Lemma 1.6, GK $\operatorname{dim}(S) \leqq 1$.

Consider the following condition on a pair of positive integers $x, y$

$$
\begin{aligned}
& f(x+i) f(x+i+j)<F(j) \text { for some } \\
& \text { integers } i>0 \text { and } j>0 \text { with } i+j \leqq y .
\end{aligned}
$$

The significance of this condition to Proposition 1.4 is given by the following result.

Lemma 1.9. Assume that (1.8) is true. Then there is a nonzero element $\beta \in S_{y}$ such that $S_{x} \beta \subseteq b S_{x+y-1}$.

Proof. Right multiplication by $S_{j}$ on the right module $V$ defines a $k$-linear map

$$
\rho: S_{j} \rightarrow \operatorname{Hom}_{k}\left(V_{x+i}, V_{x+i+j}\right) .
$$


If (1.8) holds, then the kernel of $\rho$ is not zero, and if $0 \neq w \in \operatorname{ker}(\rho)$, then $S_{x+i} w \subseteq b S_{x+i+j-1}$. Thus, $\beta=u w v \in S_{y}$ has the required property, for any nonzero elements $u \in S_{i}$ and $v \in S_{y-i-j}$.

The rest of the proof of Proposition 1.4 uses estimates which can be made for any real valued function $f(n)$ defined on $\mathbb{N}$, such that $f(n) \geqq 1$ for all $n$. So we fix such a function, and set $F(n)=f(0)+\cdots+f(n)$.

Lemma 1.10. There exists a constant $c_{1}>0$ such that, if (1.8) is false for all integers $x, y \geqq 0$, then $F(x+y)-F(x) \geqq c_{1} y^{3 / 2}$.

Proof. The hypothesis is that $f(x+i) f(x+i+j) \geqq F(j)$ for all integers $i, j>0$ with $i+j \leqq y$. If $y=0$ or $y=1$, when (1.8) is vacuously false, the desired equation holds for any $c_{1} \leqq 1$. If $y>1$, we expand

$$
\begin{aligned}
(F(x+y)-F(x))^{2} & =(f(x+1)+\cdots+f(x+y))^{2} \\
& =\sum_{j=1}^{y} f(x+j)^{2}+2 \sum_{\substack{i,>>0 \\
t+1 \leqq}} f(x+i) f(x+i+j) \\
& \geqq 2 \sum_{\substack{i,>0 \\
i+1 \leqq}} F(j) \geqq 2 \sum_{\substack{i,>>0 \\
i+1 \leqq y}}(j+1) \\
& =2 \sum_{j=1}^{y-1}(y-j)(j+1) \geqq c_{2} y^{3},
\end{aligned}
$$

for the appropriate constant $c_{2}>0$ independent of $y$.

Lemma 1.11. Let $y_{0}, y_{1}, \ldots$ be a sequence of positive integers. Set $x_{0}=0$ and $x_{n}=y_{0}+\cdots+y_{n-1}$ for $n>0$. Suppose that $F(n) \leqq c n^{r}$ for some constants $c>0$ and $0<r<6 / 5$. Assume moreover that (1.8) is false for every $n>0$, when $x=x_{n}$ and $y=y_{n}$. Then there exist constants $d>0$ and $0<s<5 / 3$ such that $x_{n} \leqq d n^{s}$ for all $n>0$.

Proof. By the previous lemma,

$$
F\left(x_{n+1}\right)>F\left(x_{n+1}\right)-F(0)=\sum_{i=0}^{n}\left(F\left(x_{i+1}\right)-F\left(x_{i}\right)\right) \geqq \sum_{i=0}^{n} c_{1} y_{i}^{\frac{3}{2}} .
$$

We use Hölder's inequality

$$
\left(\sum_{i=0}^{n} y_{i}^{p}\right)^{\frac{1}{p}}\left(\sum_{i=0}^{n} z_{t}^{q}\right)^{\frac{1}{q}} \geqq \sum_{l=0}^{n} y_{i} z_{i}, \quad 1 / p+1 / q=1 .
$$

Setting $z_{i}=1$ and taking $p$-th powers yields $(n+1)^{p-1} \sum_{i=0}^{n} y_{i}^{p} \geqq\left(\sum_{i=0}^{n} y_{i}\right)^{p}$. Thus

$$
(n+1)^{\frac{1}{2}} \sum_{i=0}^{n} y_{i}^{\frac{3}{2}} \geqq\left(\sum_{i=0}^{n} y_{i}\right)^{\frac{3}{2}}=x_{n+1}^{\frac{3}{2}} .
$$


Combining the hypothesis on the growth of $F$ with (1.11.1) and (1.11.2) yields a constant $c_{3}>0$ such that

$$
c x_{n+1}^{r} \geqq F\left(x_{n+1}\right) \geqq c_{3}(n+1)^{-\frac{1}{2}} x_{n+1}^{\frac{3}{2}},
$$

for all $n \geqq 0$. Thus, there exists $d>0$ such that $x_{n} \leqq d n^{s}$, where $s=$ $(3-2 r)^{-1}<5 / 3$, as required.

Lemma 1.12. Assume that there exist constants $c>0$ and $0<r<3 / 2$ such that $F(n) \leqq c n^{r}$ for all $n \geqq 0$. For any $x,(1.8)$ is true if $y$ is sufficiently large. On the other hand, (1.8) fails when $y=1$.

Proof. If (1.8) is false then Lemma 1.10 implies that $c(x+y)^{r} \geqq F(x+y) \geqq$ $c_{1} y^{3 / 2}$, giving the required contradiction. The final assertion is vacuously true.

Lemma 1.13. Assume that there exists constants $c>0$ and $0<r<6 / 5$ such that $F(n) \leqq c n^{r}$ for all $n \geqq 0$. Then there are sequences of strictly positive integers $\omega_{n}$ and $\tau_{n}=\omega_{0}+\cdots+\omega_{n-1}$ such that:

(i) For every $n>0,(1.8)$ is true for $x=\tau_{n}$ and $y=\omega_{n}$;

(ii) For some positive constants $d>0$ and $0<s<5 / 3$ we have $\tau_{n} \leqq$ $d n^{s}$, for all $n>0$.

Proof. We define sequences $y_{m}$ and $x_{m}=y_{0}+\cdots+y_{m-1}$ as follows: $y_{m}=1$ if $m$ is even, and if $m$ is odd, then $y_{m}$ is the largest integer so that (1.8) fails with $x=x_{m}$ and $y=y_{m}$. This integer exists by Lemma 1.12. We set $\omega_{0}=y_{0}=1$ and $\omega_{n}=y_{2 n-1}+1=y_{2 n-1}+y_{2 n}$ if $n>0$, so that $\tau_{n}=y_{0}+\cdots+y_{2 n-2}=x_{2 n-1}$. Then (1.8) holds for $x=\tau_{n}=x_{2 n-1}$ and $y=\omega_{n}=y_{2 n-1}+1$ because of the maximality of $y_{2 n-1}$. The bounds given in Lemma 1.11 apply to the sequence $x_{m}$, and with a change of the constant $d$, they carry over to $\tau_{n}$.

Proof of Proposition 1.4. Recall that $F(n)=\operatorname{dim} S_{n}$ and so Lemma 1.6 implies that there exist constants $c>0$ and $r<6 / 5$ such that $F(n) \leqq c n^{r}$ for all $n \geqq 0$. Thus, Lemma 1.13 applies and we define the $\left\{\tau_{n}\right\}$ by that result; thus Proposition 1.4(i) is satisfied. As $\tau_{n}+\omega_{n}=\tau_{n+1}$, Proposition 1.4(ii) follows from Lemma 1.9.

Of course, this also completes the proof of Corollary 1.5; in other words, if $R$ is a domain in Theorem 1.1, then $G K \operatorname{dim}(D) \leqq 1$. The next step is to prove that, in this case, $D$ is a finitely generated division ring. Despite the fact that $R$ is finitely generated, this does not seem to be quite obvious, as the following example shows:

Example. Let $K$ be the field extension of $k$ generated by the elements $x^{1 / 2^{n}}, n \in$ $\mathbb{N}$, and let $\sigma$ be the automorphism of $K$ defined by $\sigma\left(x^{1 / 2^{n}}\right)=x^{1 / 2^{n+1}}$. Let $A$ be the graded subring of $K[z ; \sigma]$ generated by $z$ and $z x$. Then $x^{1 / 2^{n}}=z^{n} x z^{-n}$. It follows that the graded division ring generated by $A$ is $K\left[z, z^{-1} ; \sigma\right]$. 
Fortunately, this is not a conterexample to Theorem 1.1, since, as is wellknown, $A$ is a free ring.

The next lemma follows from [St, Lemma 1.4].

Lemma 1.14. Let $A \subset B$ be $k$-algebras such that $B / A$ is finite dimensional. Then, $A$ is right Noetherian, respectively finitely generated, if and only if $B$ is right Noetherian, respectively finitely generated.

Theorem 1.15. Let $R$ be a finitely graded $k$-algebra and assume that $R$ is an Ore domain with graded quotient division ring $Q(R)=D\left[z, z^{-1} ; \sigma\right]$. Then, as a division ring, $D$ is finitely generated over $k$.

Proof. By regrading $R$, if necessary, we may assume that $z \in Q(R)_{1}$. Also, since $R_{0}$ is finite dimensional, Lemma 1.14 implies that $R^{\prime}=k \oplus \bigoplus_{i>0} R_{i}$ is still a finitely graded Ore domain with $Q\left(R^{\prime}\right)=Q(R)$. Thus, we may assume that $R_{0}=k$. Let $R$ be generated as a $k$-algebra by homogeneous elements $\left\{r_{l} \in R_{m_{l}}: 1 \leqq i \leqq n\right\}$ and let $T$ be the $k$-algebra generated by $\left\{z, r_{1} z^{-m_{1}+1}, \ldots, r_{n} z^{-m_{n}+1}\right\}$. Clearly, $T$ is a finitely graded domain, generated in degree one and containing $R$. If $t_{1}, t_{2}$ are non-zero homogeneous elements of $T$, then $t_{1} T \cap t_{2} T \supseteq t_{1} R \cap t_{2} R \neq 0$ and so $T$ is still an Ore domain. Thus, by replacing $R$ by $T$, we may assume that $R$ is generated by $R_{1}$ and that $z \in R_{1}$.

Define $V_{n}=R_{n} z^{-n}$ for $n \geqq 0$ and set $V=V_{1}$. The fact that $R$ is generated in degree one implies that $R_{t+1}=R_{t} R_{1}$ for all $i \geqq 1$ and hence the $V_{l} V^{\sigma^{l}}=$ $V_{1+1}$. For $n \geqq 1$, write $D_{n}=k\left(V_{1}+\cdots+V_{n}\right)$, for the subdivision ring of $D$ generated by $V_{1}, \ldots, V_{n}$. Note that $D_{1} \subseteq D_{2} \subseteq \cdots$, and so, by its construction, $D=\bigcup D_{n}$. Also, $1 \in V$ because $z \in R_{1}$, and so $V^{\sigma^{\prime}} \subseteq V_{n}$ for $0 \leqq i \leqq n-1$. Hence

$$
D_{n}=k\left(V_{n}\right)=k\left(V+V^{\sigma}+\cdots+V^{\sigma^{n-1}}\right) .
$$

Suppose that $V^{\sigma^{m}} \nsubseteq D_{m}$, for some $m \gg 1$. Equivalently, there exists $\propto \in$ $V^{\sigma^{m}}$ which is not in $D_{m}$. Since $D_{m}$ is a division ring, this implies that $D_{m} \cap$ $D_{m} \alpha=0$, and hence that $V_{m} \cap V_{m} \alpha=0$. But, $\alpha z^{m+1}=z^{m} \sigma^{-m}(\alpha) z=z^{m} r$, where $r=\sigma^{-m}(\alpha) z \in V z=R_{1}$. Thus,

$$
0=V_{m} z^{m+1} \cap V_{m} \alpha z^{m+1}=R_{m} z \cap R_{m} r .
$$

On the other hand, writing a basis for $R_{1} z^{-1}$ over a common left denominator provides $s \in R_{n}$, for some $n$, such that $R_{1} z^{-1} \subseteq s^{-1} R_{n}$. Thus, for all $m>n$, $\left(z^{m-n} s\right) R_{1} \subseteq R_{m} z$. This contradicts the last displayed equation.

Thus, there exists $n_{0} \geqq 1$ such that $V^{\sigma^{\prime \prime}} \subset D_{n}$, for all $n \geqq n_{0}$. By (1.15.1), $D_{n+1}=D_{n}$ for all such $n$. In other words, $D=\bigcup D_{i}=D_{n_{0}}$ and so $D$ is finitely generated.

In order to prove Theorem 1.1 for non-domains, we will need the following graded analogue of the Faith-Utumi Theorem.

Lemma 1.16. Let $R$ be a prime graded, right Goldie ring that contains a regular element of positive degree, and write $Q(R)=M_{n}(\Delta)$, for some graded 
division ring $\Delta$. Then, possibly after changing the choice of $\Delta$, there exists a graded Ore domain $T \subseteq R$ such that $Q(T)=A$.

Proof. The proof of the Faith-Utumi Theorem as given in [MR, 3.2.6] carries over to this graded situation. This provides a graded Ore domain $U \subset R$, possibly without a unit, such that $Q(U)$ is conjugate to $\Delta$. Now take $T=k+U$.

We are now ready to put the pieces together in order to complete the proof.

Proof of Theorem 1.1. Let $T$ be the ring defined by Lemma 1.16. We first need to modify $T$ to make it finitely generated. Thus, assume that $R$ is generated, as a $k$-algebra, by matrices $\left\{\left(a_{i j}^{\prime}\right): 1 \leqq t \leqq n\right\}$, where each $a_{i j}^{\prime}$ is a homogeneous element of $\Delta$. Pick a non-zero homogeneous element $t \in T$ such that $a_{i j}^{\prime} t \in T$, for all $i, j, \ell$ and let $T^{\prime}$ be the $k$-algebra generated by $\left\{a_{i j}^{\prime} t, t\right\}$. Since $T^{\prime} \subseteq T \subseteq$ $R, G K \operatorname{dim}\left(T^{\prime}\right)<\frac{11}{5}$ and so $T^{\prime}$ is an Ore domain. By construction, $Q\left(T^{\prime}\right)=\Delta$ and so, by replacing $T$ by $T^{\prime}$, we may assume that $T$ is finitely generated and hence finitely graded.

By Corollary 1.5 applied to $T, G K \operatorname{dim}(D) \leqq 1$ and, by Theorem $1.15, D$ is finitely generated as a division ring over $k$. Thus, there exists a finitely generated, (ungraded) subring $U$ of $D$ with quotient field $D$. Clearly, $G K \operatorname{dim}(U) \leqq$ $G K \operatorname{dim}(D) \leqq 1$. By the theorem of Small and Warfield [SW], $U$ is a finite module over its centre $Z(U)$ and $Z(U)$ is a finitely generated $k$-algebra of dimension one. Thus $D$ is finite over its centre, and its centre $K$ is a function field in one variable.

Finally, if $k$ is algebraically closed, then the Brauer group of $K$ is zero by Tsen's Theorem [Co, p. 374]. Hence $D=K$.

Remark 1.17. We note that Theorem 1.1 generalizes routinely to semiprime rings. Indeed, let $S$ be finitely graded, semiprime Goldie ring with $G K \operatorname{dim}(S)$ $<\frac{11}{5}$. Then the minimal prime ideas $\left\{P_{i}\right\}$ of $S$ are graded and $S \hookrightarrow \bigoplus S / P_{l}$. Thus, by Theorem 1.1, $Q(S)=\bigoplus Q\left(S / P_{l}\right)=\bigoplus M_{n_{t}}\left(D_{i}\left[z_{i}, z_{l}^{-1} ; \sigma_{l}\right]\right)$, where each $D_{t}$ is a finite module over its centre and $Z\left(D_{i}\right)$ is a finitely generated field extension of $k$ of transcendence degree $\leqq 1$.

Proof of Theorem 1.2. Let $X$ denote the non-singular projective model of the function field $K$, and let $g$ be the genus of $X$. Since $K$ and $X$ are uniquely determined by $\Delta$ or by the shifted matrix algebra over $\Delta$, the automorphism $\sigma$ determines an automorphism of $X$ which we also denote by $\sigma$.

We will first treat the case that $D=K$ is itself a function field in one variable. So we assume given a finitely graded subring $R$ of $K[z ; \sigma]$, where $\sigma$ is an automorphism of $K$. We may assume that $R_{0}=k$. We choose elements $1=\alpha_{0}, \alpha_{1}, \ldots, \alpha_{r} \in K$ and $d_{i} \in \mathbb{N}$ such that the elements $\left\{a_{i}=\alpha_{l} z^{d_{i}}: 1 \leqq i \leqq\right.$ $r$ \} generate $R$ as an algebra. Let $R^{\prime}$ denote the graded $k$-algebra generated by the elements $b_{i}=\alpha_{i} z$. Since $R \subseteq R^{\prime}$, we may replace $R$ by $R^{\prime}$ and so assume 
that $R$ is generated by $R_{1}$. As in the proof of Theorem 1.15 , we set $V_{n}=R_{n} z^{-n}$ and $V=V_{1}$; thus $V_{n}=V V^{\sigma} \ldots V^{\sigma^{n-1}}$.

Let $D$ be a divisor on $X$ which is an upper bound for the polar divisors $(v)_{\infty}, v \in V$. Then the poles of the elements of $V V^{\sigma} \cdots V^{\sigma^{n-1}}$, hence of $V_{n}$, are bounded by the divisor $D_{n}=D+\sigma^{-1} D+\cdots+\sigma^{-(n-1)} D$, and the degree of this divisor is $n \delta$, where $\delta=\operatorname{deg} D$. Thus $V_{n}$ is a subspace of the space $H^{0}\left(X, C\left(D_{n}\right)\right)$ of functions with pole bounded by $D_{n}$. Since $X$ is an algebraic curve, the Riemann-Roch theorem asserts that the space of all functions with pole of order $s$ has dimension $s-g+1$, whenever $s \geqq 2 g-2$. Thus $\operatorname{dim}\left(H^{0}\left(X, \mathcal{O}\left(D_{n}\right)\right)\right)=n \delta-g+1$ for large $n$.

We now turn to the general case; thus $A=S\left[z, z^{-1} ; \sigma\right]$ is a graded simple Artinian ring, where $S$ is a simple Artinian ring that is finite over its centre $K$, and $R$ is a finitely graded subalgebra of $S\left[z, z^{-1} ; \sigma\right]$. Then $S \otimes_{K} S^{\text {op }} \cong S^{\prime}=$ $M_{r}(K)$, for some integer $r$. The graded structure and automorphisms $\sigma$ of $S$ extend naturally to $S^{\prime}$ by defining $S_{n}^{\prime}=\sum_{i+j=n} S_{i} \otimes S_{j}^{\text {op }}$ and $\sigma(a \otimes b)=\sigma(a) \otimes$ $\sigma(b)$. Thus, we may regard $R$ as a subalgebra of $S^{\prime}$. The given matrix units $\left\{e_{i j}\right\}$ of $S^{\prime}$ are necessarily homogeneous, say $e_{l j} \in S_{a(t, j)}^{\prime}$. Set $r=\max \{-a(i, j), 0\}$ and $U=k \oplus \bigoplus_{i \geq 1+r} R_{i}$. By Lemma 1.14,U is still finitely generated and hence finitely graded as a $k$-algebra and so it suffices to prove the result for $U$. If $U$ is generated as a $k$-algebra by elements $\left\{u_{i j}^{\prime} e_{1 j}\right\}$, then the choice of $r$ ensures that each $u_{i j}^{\prime} \in \Delta^{\prime}=K\left[z, z^{-1} ; \sigma\right]_{n}$ for $n=n(i, j, \ell)>0$. Thus, if $W$ is the subalgebra of $A^{\prime}$ generated by the $\left\{u_{i j}^{\prime}\right\}$, then $\operatorname{dim} W_{n}$ is quadratic by the first part of the proof. Since $U \subseteq M_{r}(W)$, the algebra $U$ also has quadratic growth.

We end this section with the examples promised earlier, which delineate the hypotheses of Theorems 1.1 and 1.2. The first shows that the Goldie condition is a necessary restriction of Theorem 1.1 .

Example 1.18. Let $R$ be the ring with generators $x, y$ and these relations: $x^{2}, x y^{m} x$, for all $m \neq 2^{n}$, and $x y^{2^{m(1)}} x \cdots y^{2^{m(r)}} x$ for all $m(i)$ such that $\sum_{i=1}^{r} m(i)$ $<r 2^{r}$. Then, $R$ is a finitely graded, prime ring with $G K \operatorname{dim}(R)=2$ such that $R$ is not Goldie.

Proof. This is similar to the example of [IS], so most of the details will be left to the reader. It is not difficult to check that $R$ has basis $\left\{y^{u}\right\} \cup\left\{y^{u} x y^{v}\right\}$ together with

$$
\begin{gathered}
\left\{y^{a} x y^{2^{m(1)}} x \cdots y^{2^{m(r)}} x y^{b}: a, b \geqq 0, m(i)>0\right. \text { with } \\
\left.\sum_{t=1+s}^{s+t} m(i) \geqq t 2^{\prime} \text { for } 0 \leqq s, t .\right\}
\end{gathered}
$$

The fact that $\sum m(i)$ is forced to be so large ensures that $G K \operatorname{dim}(R)=2$. If $a, b \in R$, then $a y^{t} b \neq 0$, for appropriate large $t$ and so $R$ is prime. Finally, $R$ is not (right) Goldie since $x R \cap y R=0$ yet $y$ is regular. 
Example 1.19. Let $\mathbb{C}(u, w)$ be the field of rational functions in two variables, with automorphism $\sigma$ defined by $\sigma(u)=u+1$ and $\sigma(w)=w$.

(i) Set $\Delta=\mathbb{C}(u)\left[z, z^{-1} ; \sigma\right]$. Then, $S=\mathbb{C}\left\langle u^{-1}, z\right\rangle$ is a graded subring of $\Delta$ with $G K \operatorname{dim}(S)=3$.

(ii) If $T=\mathbb{C}\langle u z, z, w\rangle \subset \mathbb{C}(u)[w]\left[z, z^{-1} ; \sigma\right]$, then $T$ is a finitely graded, Noetherian domain with $G K \operatorname{dim}(T)=3$. However, $Q(T) \cong E\left[w, w^{-1}\right]$, where $E$ is a division ring with $G K \operatorname{dim}(E)=\infty$.

(iii) Let $\tau$ be the automorphism of $\mathbb{C}(u, w)$ defined by $\tau(u)=u w$ and $\tau(w)=u w^{2}$ and set $U=\mathbb{C}\langle u z, w z, z\rangle \subset \mathbb{C}(u, w)\left[z, z^{-1} ; \sigma\right]$. Then, $G K \operatorname{dim}(U)$ $=\infty$.

Proof. These examples are variants of well-known results, and so most of the details will be left to the reader. Since $\left[u z^{-1}, z\right]=1$, the ring $\mathbb{C}\left\langle u z^{-1}, z\right\rangle$ is isomorphic to the first Weyl algebra. Part (i) therefore follows by mimicking the proof of [KL, Example 4.10], while part (ii) follows from [ML]. Finally, part (iii) follows from the fact that $z^{\prime} u=u^{a(t)} w^{b(t)}$, where $a(i)$ and $b(i)$ grow exponentially with $i$. This example is based on the fact that the group $G=\left\langle u, w, z \mid u w u^{-1} w^{-1}, u w z u^{-1} z^{-1}, u w^{2} z w^{-1} z^{-1}\right\rangle$ is a solvable group with no nilpotent subgroups of finite index and, hence, $G$ has exponential growth [KL, Theorem 11.9].

Parts (ii) and (iii) of Example 1.19 show that the natural generalizations of Theorems 1.1 and 1.2 to rings of Gelfand-Kirillov dimension 3, respectively surfaces, will fail. The signficance of Example 1.19(i) is that Theorem 1.2 is false if one assumes that $R$ is merely a finitely generated graded, rather than finitely graded subalgebra of $A$. Indeed, using a proof similar to that of Theorem 1.2, one can even prove:

Proposition 1.20. Let $F$ be a function field in one variable, and $\sigma$ an automorphism of $F$ of infinite order. Then, $G K \operatorname{dim}(F[z ; \sigma]) \geqq 3$.

We omit the proof of this result. This instability in the Gelfand-Kirillov dimension of related algebras illustrates the delicacy inherent to any proof of Theorems 1.1 and 1.2.

\section{The associated divisors}

Let $R$ be an finitely graded domain with $G K \operatorname{dim}(R)=2$ and assume that $R_{0}=$ $k$ is an algebraically closed field $k$. By Theorem $1.1, Q(R)=K\left[z, z^{-1} ; \sigma\right]$, where $K$ is the field of rational functions $k(X)$ of a smooth, projective curve $X$. In this section we translate the basic properties of $R$ into data concerning divisors over $X$. While these divisors cannot tell one everything about $R$, in particular they do not distinguish between rings associated to $X$ and those associated to singular curves birational to $X$, they do provide a first approximation. This will be used in the subsequent sections to elaborate on the properties of $R$. Unfortunately, if $k$ is not algebraically closed, then $Q(R)=D\left[z, z^{-1} ; \sigma\right]$, where 
$D$ is a finite dimensional division ring over $K$. The analysis of this situation is more complicated. We hope to deal with this more general case, and with the case of prime rings, in the future.

Henceforth, we use the following notation; it is slightly different from that of Section 1, since we wish to emphasize the geometry rather than the algebra.

Let $k$ be any field and $K / k$ a function field in one variable, such that $k$ is algebraically closed in $K$. Let $K / k$ have associated smooth projective model $X$, and let $(" X$ denote the structure sheaf of $X$. Geometric notation will for the most part be standard as, for example, given in [Ha]. Let $V$ be a finite dimensional nonzero subspace of $K$. By the divisor of $V$ we mean the smallest (Weil) divisor $D=D(V)$ on $X$ such that $(f)+D \geqq 0$ for all nonzero elements $f \in V$. Equivalently, $D$ is the divisor whose associated invertible sheaf $\mathcal{C}_{X}(D)$ is the coherent subsheaf $V C_{X}$ of the constant sheaf $K$ generated by $V$. Given divisors $P$ and $Q$ on $X$, we will frequently write $P \cup Q$ for the smallest divisor $\geqq P, Q$ and $P \cap Q$ for the largest divisor $\leqq P, Q$. If $\mathscr{I}$ is a subset of $X$, we write $\left.E\right|_{\text {\& }}$ for the restriction of a divisor $E$ to $\mathscr{I}$; that is, $E=\left.E\right|_{4}+E^{\prime}$, where $E^{\prime}$ is supported on $X \backslash \mathscr{I}$.

Fix a $k$-automorphism $\sigma$ of $K$ and let $\sigma$ also denote the induced automorphism of $X$. We adopt the convention that $\sigma$ acts on the right on $K$, and on the left on $X$; thus $f^{\sigma}(x)=f(\sigma(x))$ for $f \in K$ and $x \in X$. Similarly, $\sigma(E)=\sum_{t} r_{l} \sigma\left(p_{l}\right)$ for a divisor $E=\sum_{l} r_{i} p_{l}$. We will write $\bar{F}^{\sigma}$ for the inverse image $\sigma^{*} \mathscr{F}$ of a coherent sheaf $\mathscr{F}$ along $\sigma$. Note that this implies that, if $D$ is a divisor on $X$, then $C_{X}(D)^{\sigma}=C_{X}\left(\sigma^{-1} D\right)$ while, if $V \subset K$ is a finite dimensional $k$-subspace, then $D\left(V^{\sigma}\right)=\sigma^{-1} D(V)$. Finally, a graded $k$-algebra $R$ is called connected graded if $R=k+\bigoplus_{i \geqq 1} R_{l}$ is a graded ring with $\operatorname{dim}_{k} R_{l}<\infty$ for all $i$. Note that, if $A$ is a finitely graded subalgebra of $K[z ; \sigma]$, then $A_{0}$ is a finite $k$-submodule of $K$ and so $A_{0}=k$ and so $A$ is automatically connected graded.

Hypothesis 2.1. $R=k+\bigoplus_{1 \geq 1} R_{l}$ is a connected graded subalgebra of $K[z ; \sigma]$, where $z \in R_{1}, Q(R)=K\left[z, z^{-1} ; \sigma\right]$, and $\operatorname{dim}_{k}\left(R_{i}\right)<\infty$, for all $i$.

We remark that the assumption that $z \in R_{1}$, amounts to assuming that $R_{1} \neq 0$. This condition will simplify the proofs in this section but is not required in the main results of the paper, since Proposition 2.21 will provide a technique for reducing to this situation. Note that we have not assumed that $R$ is finitely generated in (2.1), because it will be more convenient to make assumptions on the sequence of divisors which is defined below.

We introduce the following standard notation. For $i \geqq 0$, write $R_{i}=\bar{R}_{i} z^{l}$. Thus $\bar{R}_{i} \subset K$ and $1 \in \bar{R}_{i}$ for all $i \geqq 0$. For each $n, D_{n}^{R}=D\left(\bar{R}_{n}\right)$ will denote the divisor defined by the space $\bar{R}_{n}$. Note that the multiplication rule $R_{t} R_{\jmath} \subseteq R_{i+j}$ translates to

$$
\bar{R}_{i} \bar{R}_{j}^{\sigma^{t}} \subseteq \bar{R}_{i+j}, \quad \text { for all } i, j \geqq 0
$$

Hence

$$
D_{i}^{R}+\sigma^{-1} D_{j}^{R} \leqq D_{i+j}^{R}
$$


The basic aim of this section is to describe the asymptotic structure of the divisor sequence $\left\{D_{n}^{R}: n \geqq 0\right\}$ associated to a ring $R$ satisfying the hypotheses of (2.1). In fact, the ring $R$ is largely irrelevant to our discussion, so we will study sequences of divisors satisfying the appropriate properties.

The appropriate condition is as follows: Define a sequence of divisors $\left\{E_{n}\right.$ : $n \geqq 0\}$ to be a $\sigma$-divisor sequence if

(2.2.1) $0=E_{0} \leqq E_{1}, E_{n}>0$ for some $n$, and

(2.2.2) There exists an integer $r$ such that $E_{n}=\bigcup_{I=1}^{r}\left(E_{i}+\sigma^{-i} E_{n-i}\right)$ for all $n \geqq 0$.

In (2.2.2) and elsewhere, we have adopted the convention that terms involving undefined divisors are to be ignored. Thus, since $E_{k}$ is undefined for $k<0$ (2.2.2) must be read as

$$
E_{n}=\bigcup_{i=1}^{\min \{n, r\}}\left(E_{i}+\sigma^{-i} E_{n-i}\right) .
$$

Lemma 2.3. (i) Let $R$ be a finitely graded k-algebra satisfying (2.1). Then $\left\{D_{n}^{R}\right\}$ is a $\sigma$-divisor sequence.

(ii) A sequence of divisors $\left\{E_{n}\right\}$ is a $\sigma$-divisor sequence if and only if $\left\{E_{n \mid, \mathscr{G}}\right\}$ is a $\sigma$-divisor sequence for each orbit $\mathscr{I}$ of $\sigma$.

Proof. (i) The first property, (2.2.1), follows from the fact that $1 \in \bar{R}_{1}$ and that $Q(R)_{0}=K$. If $R$ is generated by $\bigoplus_{l=1}^{r} R_{l}$ as a $k$-algebra, then $\bar{R}_{n}=\sum_{i=0}^{r} \bar{R}_{i} \bar{R}_{n-l}^{\sigma^{t}}$ for all $n>r$. This implies (2.2.2). The second part of the lemma is a triviality.

Lemma 2.4. Let $E_{n}$ be a $\sigma$-divisor sequence. Then the following hold for all positive integers $m, n$.

(i) $E_{m}+\sigma^{-m} E_{n} \leqq E_{m+n}$.

(ii) $0 \leqq E_{n} \leqq E_{n+1}$, and $\sigma^{-1} E_{n} \leqq E_{n+1}$.

(iii) $E_{n}=\bigcup_{i=1}^{r}\left(E_{n-i}+\sigma^{-(n-i)} E_{i}\right)$.

Moreover, (iii) is equivalent with (2.2.2).

Proof. By (2.2.2), the first assertion is true for $1 \leqq m \leqq r$. For $m>r$, we may assume by induction that $E_{m+n-l} \geqq E_{m-i}+\sigma^{-(m-l)} E_{n}$, for $1 \leqq i \leqq r$. Then

$$
E_{m+n}=\bigcup_{i=1}^{r}\left(E_{i}+\sigma^{-i} E_{m+n-i}\right) \geqq \bigcup_{i=1}^{r}\left(E_{i}+\sigma^{-i} E_{m-i}+\sigma^{-m} E_{n}\right)=E_{m}+\sigma^{-m} E_{n} .
$$

Thus, part (i) holds. Part (iii) follows by a similar induction. Part (ii) follows from part (i) and (2.2.1).

The ring described in the next example has been discussed in detail in [SZ]. We will use it to illustrate a number of the results of this paper, since its properties are in marked contrast to those of a commutative graded ring. 
Example 2. $\leqq$. Let $K=k(u)$ be a rational function field, and define $\sigma$ by $u^{\sigma}=u /(1+u)$. For $n \geqq 0$, let $p_{n}$ denote the pole of $u^{\sigma^{\prime \prime}}=u /(1+n u)$ in $X=\mathbb{P}^{1}$; thus $p_{n}=\sigma^{-n} p_{0}$. Set $U=k\langle z, u z\rangle \subset K[z ; \sigma]$. Then, $U=$ $k\{x, y\} /\left(x y-y x-x^{2}\right)$, under the identification $y=z$ and $x=u z$. The ring that interests us is $A=k+U y$. By [SZ, Lemma 2.2], $A=k\langle y, x y\rangle=k\left\langle z, u z^{2}\right\rangle$. Now, $A_{n}$ has basis $\left\{x^{t} y^{n-i}: 0 \leqq i<n\right\}$, and $x^{l}=(u z)^{\prime}=u u^{\sigma} \cdots u^{\sigma^{\prime-1}} y^{t}$ for all $i \geqq 1$. It follows that $\bar{A}_{n}$ has basis $\left\{1, u u^{\sigma} \cdots u^{\sigma^{\prime}}: 0 \leqq r \leqq n-2\right\}$ for $n \geqq 2$. Setting $D_{n}=D_{n}^{A}$, we have $D_{1}=0$ and $D_{n}=p_{0}+p_{1}+\cdots+p_{n-2}$ for $n \geqq 2$.

By [SZ, Theorem 2.3], $A$ is Noetherian if and only if $\operatorname{char}(k)=0$. As will be seen, this dichotomy is caused by the behaviour of the sequence of points $\left\{p_{n}\right\}$ : If $\operatorname{char}(k)=0$, then $|\sigma|=\infty$ and the $p_{n}$ are distinct, but if, for example, $\operatorname{char}(k)=2$, then $|\sigma|=2$ and $p_{n}=p_{n+2}$ for all $n$. In this case, $D_{2 r+1}=r p_{0}+r p_{1}$ and $D_{2(r+2)}=(r+1) p_{0}+r p_{1}$, for all $r \geqq 0$.

As this example illustrates, the structure of the finitely graded domain $R$ depends crucially on the order of $\sigma$, and we will deal with the cases of finite and infinite order separately. We begin with the case of finite order.

Proposition 2.6. Let $\left\{D_{n}\right\}$ be a $\sigma$-divisor sequence. Assume that $|\sigma|=d<\infty$ and write $\Delta_{n}=D_{n}-D_{n-1}$, for $n \geqq 1$. Then:

(i) $A_{n} \geqq 0$ for all $n \geqq 1$ and is a periodic function of $n$ for $n \gg 0$.

(ii) Let $\Delta_{n}$ have period $k_{0}$ for $n \geqq n_{0}$. Set $k=d k_{0}$ and $Z=D_{n_{0}+k}-D_{n_{0}}$; thus $D_{n+k}=D_{n}+Z$ for all $n \geqq n_{0}$. Then $Z$ is $\sigma$-invariant and $D_{m k} \leqq m Z$ for all $m$.

Proof. (i) It follows from (2.2.1) and Lemma 2.4(ii) that $D_{n}$ increases with $n$, hence that $A_{n} \geqq 0$. Consequently, Lemma 2.4(iii) implies that

$$
D_{n}=\bigcup_{i=1}^{r}\left(D_{n-1}+\sigma^{-(n-1)} D_{i}\right) \leqq D_{n-1}+B, \quad \text { where } B=\bigcup_{j=0}^{d-1}\left(\sigma^{-j} D_{r}\right) .
$$

Thus $0 \leqq A_{n} \leqq B$ for all $n$, which shows that there are only finitely many possibilities for the divisors $\Delta_{n}$.

For $1 \leqq i \leqq r$ and for $n>r$, we may write $D_{n-i}=D_{n-r}+\left(\Delta_{n-l} \cdots+\right.$ $\left.A_{n-r+1}\right)$. By Lemma 2.4(iii),

$$
\begin{aligned}
A_{n}+\Delta_{n-1}+\cdots \Delta_{n-r+1} & =D_{n}-D_{n-r} \\
& =\bigcup_{i=1}^{r}\left(\Delta_{n-i}+\cdots+\Delta_{n-r+1}+\sigma^{-(n-i)} D_{i}\right) .
\end{aligned}
$$

This determines $\Delta_{n}$ in terms of the data consisting of the $r$ previous differences $\Delta_{j}$ for $n-r+1 \leqq j \leqq n-1$, the divisors $D_{i}$ for $1 \leqq i \leqq r$, and the congruence class of $n$ (modulo $d$ ). Since there are finitely many possibilities for this data, $\Delta_{n}$ is periodic for large $n$.

(ii) Since $A_{i}$ is periodic, $Z=\Delta_{n+1}+\cdots+\Delta_{n+k}$, and so $D_{n+m k}=D_{n}+m Z$ for all $n \geqq n_{0}$ and all $m$. Also, Lemma 2.4(ii) implies that $\sigma^{-1} D_{t} \leqq D_{t+1}=$ 
$D_{t}+\Delta_{t+1}$, for any $t$. Setting $t=n+m k$, we find that $m\left(\sigma^{-1} Z-Z\right) \leqq$ $D_{n}-\sigma^{-1} D_{n}+\Delta_{t+1}$. The right side of this inequality is bounded independently of $m$, and it follows that $\left(\sigma^{-1} Z-Z\right) \leqq 0$. Since the total degrees of the two divisors are equal, $\sigma^{-1} Z=Z$.

Since $d$ divides $k$, Lemma 2.4(i) implies that $n D_{m k} \leqq D_{r m k}$ for any $n \geqq 0$. Moreover, $D_{(i+j) m k}=D_{\text {tmk }}+j m Z$, for $i \gg 0$ and $j \geqq 0$. Then $(i+j) D_{m k} \leqq$ $D_{\text {lmk }}+j m Z$, for all $j$, independently of $i$ and $n$. Thus, $D_{m k} \leqq m Z$, as required.

Lemma 2.7. Let $\left\{D_{n}\right\}$ be a $\sigma$-divisor sequence. Let $\mathscr{I}$ be a subset of $X$ consisting entirely of fixed points of $\sigma$ and set $E_{n}=D_{n \mid \vartheta}$. Then there exists $\ell$ such that $E_{n \prime}=n E_{\ell}$ for all $n \geqq 1$.

Proof. By Lemma 2.3(ii), $\left\{E_{n}\right\}$ is a $\sigma$-divisor sequence, and it suffices to prove the lemma when $\mathscr{I}$ is a single fixed point $p$. For $n>r$, write $E_{n}=t_{n} p$ for integers $t_{n}$. Formula (2.2.2) implies that $t_{n}=\sup _{t=1}^{r}\left(t_{l}+t_{n-1}\right)$. Choose $1 \leqq \ell \leqq r$ such that $\lambda=t / / \ell$ is as large as possible. Then, by induction, $t_{j} \leqq \lambda j$ for all $j$ and so $t_{n t}=n t$, for all $n \geqq 1$.

The $m$ th Veronese ring of a graded ring $R$ is the ring $R^{(m)}=\bigoplus_{i \geqq 0} R_{l m}$, graded by $R_{l}^{(m)}=R_{i m}$. Since the divisor sequence of $R^{(m)}$ is $\left\{D_{n}^{R^{(m)}}=D_{m n}^{R}\right\}$, we define the $m$ th Veronese sequence of a sequence of divisors $\left\{E_{n}\right\}$ to be the sequence $\left\{E(m)_{n}=E_{m n}\right\}$. Note that, if $\left\{E_{n}\right\}$ is a $\sigma$-divisor sequence, then $E(m)_{i}+\sigma^{-i m} E(m)_{j} \leqq E(m)_{i+j}$ and so $\{E(m)\}$ satisfies (2.2.1) and (2.4)(i). However, (2.2.2) need not hold and, when this occurs, $R^{(m)}$ has unpleasant properties:

Proposition 2.8. Let $\left\{D_{n}\right\}$ be a $\sigma$-divisor sequence, where $|\sigma|=d<\infty$. Let $k$ and $Z$ be defined as in Proposition 2.6(ii). The Veronese sequence $\left\{D(k)_{n}=D_{n k}\right\}$ is a $\sigma^{k}$-divisor sequence if and only if $D_{m k}=m Z$ for large $m$.

Proof. Suppose that $\left\{D(k)_{n}\right\}$ is a $\sigma^{h}$-divisor sequence. Since $\sigma^{k}=i d$, Lemma 2.7 implies that, for some $\ell, D_{n t}=n D_{\ell}$, for all $n \geqq 1$. But, by Proposition 2.6, $W:=m Z-D_{m k}$ is independent of $m \gg 0$. Thus,

$$
W=D_{m n k t}-m n t Z=n D_{m k t}-m n t Z=n W,
$$

for all $n, m \gg 0$. Therefore, $W=0$ and $D_{m k}=m Z$ for $m \gg 0$.

Conversely, if $D_{m k}=m Z$ for $m \geqq m_{0}$, then $D_{n k}=D_{m_{0} k}+D_{\left(n-m_{0}\right) k}$, for all $n \geqq 2 m_{0}$. Thus, (2.2.2) holds with $r=2 m_{0}$, and $\left\{D(k)_{n}\right\}$ is a $\sigma^{k}$-divisor sequence.

The implications of this proposition for a finitely graded algebra are derived in Theorem 4.9.

We next consider the asymptotic structure of a $\sigma$-divisor sequence when $|\sigma|=\infty$. In this case the genus $g$ of $X$ is either zero or one [Ha, Ex. V.1.11, p. 368]. If $g=1$, then $X$ is an elliptic curve, and $\sigma$ is translation by a point 
of infinite order. All orbits of $\sigma$ are infinite in this case. If $g=0$, then $X \cong \mathbb{P}_{h}^{l}$. For an appropriate choice of coordinate function $v$, an automorphism $\sigma$ of $\mathbb{P}^{i}$ can be described by $\sigma(v)=v+1$ (the additive case) or by $\sigma(v)=\lambda v$, for some $\lambda \in k^{*}$ (the multiplicative case). Since we are assuming that $|\sigma|=\infty$, the characteristic of $k$ is zero if $\sigma$ is additive, whilst $\lambda$ is not a root of unity if $\sigma$ is multiplicative. In these cases, the finite orbits consist of the fixed points, these being the point at infinity in the additive case or the two fixed points 0 and $\infty$ in the multiplicative case.

These observations have the following important consequence:

If $|\sigma|=\infty$, then the only finite orbits of $\sigma$ are fixed points.

The reason for its importance is that the restriction of a $\sigma$-divisor sequence $\left\{D_{n}\right\}$ to a fixed point has the pleasant structure described by Lemma 2.7.

Notation 2.10. Let $\left\{D_{n}\right\}$ be a $\sigma$-divisor sequence, where $|\sigma|=\infty$. Fix an infinite orbit $\mathscr{I}=\left\{p_{i}=\sigma^{-1}\left(p_{0}\right): i \in \mathbb{Z}\right\}$ of $\sigma$ and write $E_{n}=\left.D_{n}\right|_{\mathscr{q}}=\sum_{l} t_{i}^{\prime \prime} p_{l}$. Assume that $E_{n} \neq 0$ for some $n$. Since $\sigma^{-1}\left(p_{\imath}\right)=p_{t+1},(2.2 .2)$ implies that there exists $i_{0}$ such that $t_{i}^{n}=0$ for all $i<i_{0}$ and all $n$. Reindex the orbit so that $t_{i}^{n}=0$ if $i<0$ but $t_{0}^{m} \neq 0$, for some $m$. Formula (2.4)(i) translates to

$$
t_{i}^{m}+t_{i-m}^{n} \leqq t_{i}^{m+n}
$$

and (2.2.2) and (2.4)(iii) translate to

$$
t_{t}^{n}=\max _{r=1}^{r}\left(t_{i}^{r}+t_{i-v}^{n-v^{r}}\right)=\max _{v=1}^{r}\left(t_{i}^{n-v^{r}}+t_{i-n+r}^{v}\right),
$$

for $n>r$.

Proposition 2.11. With the above notation, there exist integers $a, b, c$, with $a \geqq 0, b>0$ and $a \geqq c>-b$, and $a$ sequence of integers

$$
\begin{aligned}
0 & <\mathbf{r}_{0} \leqq \mathbf{r}_{1} \leqq \cdots \leqq \mathbf{r}_{a-1}<\mathbf{r}_{a} \\
& =\mathbf{r}_{\max }=\mathbf{s}_{-b}>\mathbf{s}_{1-b} \geqq \cdots \geqq \mathbf{s}_{c^{\prime}-1}>\mathbf{s}_{c}=0
\end{aligned}
$$

such that

(i) $\mathbf{r}_{k}+\mathbf{s}_{k} \leqq \mathbf{r}_{\max }$ for $0 \leqq k \leqq c$,

(ii) For all $n$,

$$
t_{k}^{n} \leqq \begin{cases}\mathbf{r}_{k} & \text { for } 0 \leqq k \leqq a \\ \mathbf{r}_{\max } & \text { for } a \leqq k \leqq n-b \\ \mathbf{s}_{k-n} & \text { for } n-b \leqq k \leqq n+c \\ 0 & \text { for } k \geqq n+c,\end{cases}
$$

(iii) The inequality (ii) is an equality if $n \gg 0$.

For convenience, set $\mathbf{r}_{i}=0$ for $i<0$ and $\mathbf{s}_{i}=0$ for $i \notin[-b, c]$.

Proof. We begin by constructing the integers $\mathbf{r}_{j}$. By (2.4)(ii), (2.2.2) and induction,

$$
E_{n} \leqq \bigcup_{i=1}^{r}\left(E_{r}+\sigma^{-i} E_{n-i}\right) \leqq E_{r}+\sigma^{-1} E_{r}+\cdots+\sigma^{-(n-1)} E_{r} .
$$


This inequality shows that, for all $n$ and all $i$, the integer $t_{i}^{n}$ is bounded by the total degree $\sum_{j} t_{j}^{r}$ of $E_{r}$. Set

$$
\mathbf{r}_{\max }=\max _{n, i}\left\{t_{i}^{n}\right\} \quad \text { and } \quad \mathbf{r}_{i}=\max _{n}\left\{t_{i}^{n}\right\} .
$$

The inequality (2.10.1) implies that

$$
t_{i}^{n-1} \leqq t_{i}^{n} \text { and } t_{i-1}^{n-1} \leqq t_{i}^{n} .
$$

It follows that $t_{i}^{n}$ is an increasing function of $n$, that $\mathbf{r}_{i}=t_{i}^{n}$ for $n \gg 0$, and that $\mathbf{r}_{i}$ is an increasing function of $i$. We define $a=\min \left\{i: \mathbf{r}_{i}=\mathbf{r}_{\max }\right\}$. Note that $\mathbf{r}_{0}>0$. since some $t_{0}^{m}$ is non-zero.

In order to define the integers $\mathbf{s}_{\jmath}$, set $G_{n}=\sigma^{n} E_{n}$. Then $G_{n}=\sum g_{i}^{n} p_{i}$, with $g_{i}^{n}=t_{t+n}^{n}$. By (2.10.2).

$$
g_{t}^{n}=t_{i+n}^{n}=\max _{v=1}^{r}\left(t_{i+n}^{v}+t_{i+n-v}^{n-v}\right)=\max _{v=1}^{r}\left(g_{t+n-v}^{r}+g_{t}^{n-v}\right) .
$$

This implies that $G_{n}=\bigcup_{1=i}^{r}\left(G_{n-v}+\sigma^{n-v} G_{v}\right)$, and by Lemma 2.4 that $\left\{G_{n}\right\}$ is a $\sigma^{-1}$-divisor sequence. We may repeat the analysis of the last two paragraphs for $\left\{G_{i}\right\}$, except that, since $p_{0}$ has been fixed by the data $E_{n}$, we are not free to reindex, as was done in (2.10). This analysis leads to integers $\mathbf{s}_{i}=\sup _{n}\left\{g_{i}^{n}\right\}=$ $\sup _{n}\left(t_{n+i}^{n}\right)$ such that $\mathbf{r}_{\max } \geqq \mathbf{s}_{\imath} \geqq \mathbf{s}_{i+1} \geqq 0$, for all $i$ and $\mathbf{s}_{l}=t_{n+i}^{n}$ for $n \gg 0$. We define $c$ and $b$ by $\mathbf{r}_{\max }=\mathbf{s}_{-b}>\mathbf{s}_{1-b} \geqq \cdots \geqq \mathbf{s}_{c-1}>\mathbf{s}_{c}=0$. Then the inequality of part (ii) is true and, by (2.11.1), it is an equality for $n \gg 0$.

It remains to check that $a \geqq c, b>0$ and that (i) holds. These all follow from the inequality (2.10.1). Substituting $i=j+n$ and taking $m, n \gg 0$ gives $\mathbf{s}_{j}+\mathbf{r}_{j} \leqq \mathbf{r}_{\max }$. Thus (i) holds for all values of $j$. Since $\mathbf{r}_{a}=\mathbf{r}_{\max }$, this forces $\mathbf{s}_{a}=0$ and $c \leqq a$. Similarly, since $\mathbf{r}_{0}>0, \mathbf{s}_{n}<\mathbf{r}_{\max }$ and so $b>0$.

Corollary 2.12. With the notation of (2.10) and (2.11), set $E_{n}=\left.D_{n}\right|_{\%}$. Set $\omega_{i}=\mathbf{r}_{\max }-\mathbf{r}_{i}-\mathbf{s}_{i}$ for $i \in \mathbb{Z}$ and define $\Omega_{q}=\sum_{t} \omega_{t} p_{t}=\omega_{1-b} p_{1-b}+\cdots+$ $\omega_{a-1} p_{a-1}$. Then $\Omega_{q} \geqq 0$, and $\Omega_{q}=0$ if and only if $b=1, a=c$ and $\mathbf{r}_{i}+\mathbf{s}_{i}=\mathbf{r}_{\max }$ for $0 \leqq i \leqq a$. Moreover,

$$
E_{m+n}=E_{m}+\sigma^{-m} E_{n}+\sigma^{-m} \Omega_{q} \text { for } m, n \gg 0 .
$$

and

$$
\sigma^{-s} E_{n} \geqq \sigma^{-n} \Omega_{q} \quad \text { whenever } n \gg 0 \text { and } a+b \leqq s \leqq n-a-b \text {. }
$$

This routine computation is left to the reader. The divisor $\Omega_{\mathscr{G}}$ appearing in this corollary will be called the gap divisor on the orbit $\mathscr{I}$, since its definition is precisely what is required for $(2.12 .1)$ to hold. This divisor can be non-zero; for example, this is the case if $A$ is the ring of Example 2.5, with char $k=0$ and $\mathscr{I}=\left\{p_{i}\right\}$, since then $\Omega_{\mathscr{q}}=p_{0}$. Note that this also implies that $A^{(/)}$is never generated in degree one, giving another proof of [SZ, Corollary 3.2]. 
Corollary 2.13. Let $\left\{D_{n}\right\}$ be a $\sigma$-divisor sequence, where $|\sigma|=\infty$. Then:

(i) For any $t \geqq 1$, the Veronese sequence $\left\{D(t)_{n}\right\}$ is a $\sigma^{\prime}$-divisor sequence.

(ii) If $t$ is sufficiently large, then for all $n \geqq 3$,

$$
D(\ell)_{n}=\left\{D(\ell)_{n-2}+\sigma^{-/(n-2)} D(/)_{2}\right\} \cup\left\{D(\ell)_{n-1}+\sigma^{-/(n-1)} D(/)_{1}\right\} .
$$

Proof. (i) We may verify this corollary separately for each orbit. It is true for the fixed points, by Lemma 2.7. Thus, let $E_{n}$ be the restriction of $D_{n}$ to an infinite orbit $\mathscr{I}$. As we remarked already, the formulae of Lemma 2.4(i, ii) carry over directly to a Veronese sequence. Choose $s$ large enough so that (2.12.1) holds for all $m, n \geqq s t$ and that $\sigma^{-s t} \Omega_{q} \cap \Omega_{q}=0$. It suffices to show that (2.2.2) holds for $E(t)$, with $r=3 s$. For $t \geqq r,(2.12 .1)$ implies that

$$
E_{t t}=E_{s t}+\sigma^{-s t} E_{(t-s) t}+\sigma^{-s t} \Omega_{q}=E_{2 s t}+\sigma^{-2 s t} E_{(t-2 s)}+\sigma^{-2 s t} \Omega_{\phi} .
$$

Since $\sigma^{-s t} \Omega_{q} \cap \sigma^{-2 s t} \Omega_{q}=0$, it follows that

$$
E_{t \prime}=\left(E_{s /}+\sigma^{-s t} E_{(t-s)}\right) \cup\left(E_{2 s /}+\sigma^{-2 s t} E_{(t-2 s)}\right) .
$$

This verifies (2.2.2), as required. Part (ii) is proved similarly.

Let $R$ be a finitely generated algebra that satisfies the hypotheses of (2.1), with $|\sigma|=\infty$. Then Corollary 2.13 provides a weak version of two results that will be proved later: the Veronese ring $R^{(t)}$ is finitely generated for all $t$ (Theorem 5.6), and generated in degrees one and two for $f \gg 0$ (Proposition $6.6)$.

The fixed points of $\sigma$ complicate our description somewhat, so we will frequently consider the following supplementary hypotheses:

Hypothesis 2.14. The sequence $\left\{D_{n}\right\}$ is a $\sigma$-divisor sequence, where $|\sigma|=\infty$, and for each fixed point $p$ of $\sigma$ on $X$, there exists an integer $s=s_{p}$ such that the multiplicity of $D_{n}$ on $p$ equals $s n$, if $n \gg 0$.

The analogous hypothesis for a graded ring is given by:

Hypothesis 2.15. The graded ring $R$ satisfies (2.1), $|\sigma|=\infty$, and the associated divisor sequence $\left\{D_{n}^{R}\right\}$ satisfies (2.14).

Assume that $|\sigma|=\infty$ and let $\left\{D_{n}\right\}$ be a $\sigma$-divisor sequence. By Lemma 2.7 and Corollary 2.13, Hypothesis 2.14 will be satisfied automatically for some Veronese sequence $\left\{D(\ell)_{n}\right\}$. Similar comments apply to (2.15).

If $\left\{D_{n}\right\}$ satisfies (2.14), then the gap divisor for $\left\{D_{n}\right\}$ is defined to be $\Omega=\sum \Omega_{\phi}$, the sum being taken over the infinite orbits of $\sigma$ which meet the support of $D_{n}$ for some $n$. This is well-defined, since the support of the $\left\{D_{n}\right\}$ is contained in a finite number of $\sigma$-orbits. With this definition, Corollary 2.12 implies that

$$
D_{m+n}=D_{m}+\sigma^{-m} D_{n}+\sigma^{-m} \Omega \text { for all } m, n \gg 0 .
$$

The proof of the next result is routine and is therefore omitted. 
Lemma 2.17. Assume that the sequence $\left\{D_{n}\right\}$ satisfies (2.14). Let $n_{0}$ be an integer such that the inequalities of (2.11)(ii) are equalities for $n \geqq n_{0}$ on each infinite orbit, and that (2.16) holds for $n \geqq n_{0}$. For $n \geqq n_{0}$, let $C_{n}=$ $D_{n}+\Omega$. Then

(i) $C_{i+j}=C_{t}+\sigma^{-i} C_{j}$ for $i, j \geqq n_{0}$.

(ii) $D_{i+j}=D_{i}+\sigma^{-t} C_{j}$ for $i, j \geqq n_{0}$.

Let $\left\{D_{n}, n \in \mathbb{N}\right\}$ be a sequence of non-negative divisors that $D_{m}+\sigma^{-m} D_{n}$ $\leqq D_{m+n}$ for all $m, n$, i.e., for which (2.2.1) and (2.4)(i) hold. Suppose that (2.2.2) does not hold, so that $D_{n}$ is not a $\sigma$-divisor sequence. Then either the supports of the divisors $D_{n}$ meet infinitely many orbits, or else (2.2.2) fails for the restriction of $D_{n}$ to some $\sigma$-orbit $\mathscr{T}$. The next proposition, which complements Proposition 2.11, describes this second possibility.

Proposition 2.18. Assume that $|\sigma|=\infty$. Let $\left\{D_{n}: n \geqq 0\right\}$ be a sequence of non-negative divisors whose supports are contained in a single orbit $\mathscr{I}$. Suppose that $D_{m}+\sigma^{-m} D_{n} \leqq D_{m+n}$ for all $m, n$ but that the sequence is not a $\sigma$-divisor sequence.

(i) If $\mathscr{I}=\{p\}$ where $p$ is a fixed point, write $D_{n}=s_{n} p$. Then the ratios $s_{n} / n$ do not attain a maximum.

(ii) If $\mathscr{I}$ is an infinite orbit, then the multiplicities of $D_{n}$ are unbounded.

Proof. The proof of (i) follows the lines of Lemma 2.7.

(ii) For $k \geqq 1$, define a sequence of divisors $\left\{E_{n}^{k}\right\}$ by setting $E_{1}^{k}=D_{t}$ for $1 \leqq i \leqq k$, and

$$
E_{n}^{k}=\bigcup_{i=1}^{k}\left(E_{i}^{k}+\sigma^{-i} E_{n-i}^{k}\right) \text { for } n>k .
$$

The hypotheses imply that, for each $k,\left\{E_{n}^{k}\right\}$ is a $\sigma$-divisor sequence, that $E_{n}^{k} \leqq E_{n}^{k+1}$ for all $n$, and that $D_{n}=\bigcup_{k} E_{n}^{k}$. Since $\left\{D_{n}\right\}$ is not a $\sigma$-divisor sequence, $\left\{D_{n}\right\} \neq\left\{E_{n}^{k}\right\}$, for any $k$ and so $\left\{E_{n}^{k}\right\} \neq\left\{E_{n}^{k+1}\right\}$ infinitely often. Since the inequality in Proposition 2.11(ii) is an equality for all large $n$, it follows that there exist infinitely many values of $k$ for which $E_{n}^{k}<E_{n}^{k+1}$ for all large $n$.

Now consider the data produced by Proposition 2.11 for the $\sigma$-divisor sequences $\left\{E_{n}^{k}\right\}$, with one difference; the point $p_{0} \in \mathscr{F}$ is fixed arbitrarily and independently of $k$. Let $a^{t}, b^{t}$ and $\mathbf{r}_{\max }^{t}$ be the numbers that Proposition 2.11 provides for the sequence $\left\{E_{n}^{t}\right\}$. While $a^{t}$ and $b^{t}$ depend upon the point $p_{0}$, their sum does not. Thus, Proposition 2.11 shows that, for large $n$, the total degree $\sum_{i} t_{i}^{n}$ of $E_{n}^{k}$ has these bounds:

$$
\left(n-b^{k}-a^{k}\right) \mathbf{r}_{\max }^{k} \leqq \operatorname{deg} E_{n}^{k} \leqq n \mathbf{r}_{\max }^{k} .
$$

Thus if $\ell \geqq k$ and if $\mathbf{r}_{\max }^{\prime}=\mathbf{r}_{\max }^{k}$, then $\operatorname{deg} E_{n}^{\prime} \leqq \operatorname{deg} E_{n}^{k}+\left(b^{k}+a^{k}\right) \mathbf{r}_{\max }^{k}$ for all $n \gg 0$. Combined with the conclusion of the previous paragraph, this shows that $\mathbf{r}_{\max }^{k}$ is unbounded. 
We will now discuss an analogue of the divisor sequence when we drop the assumption that $z$ is in the ring. We assume that $|\sigma|=\infty$. Let $A$ be a finitely graded subring of $K[t ; \sigma]$ such that the quotient ring $Q(A)$ has the form $K\left[t, t^{-1} ; \sigma\right]$, where $t \in Q(A)_{1}$. We set $A_{i}=\bar{A}_{i} t^{t}$ as before. Then (2.1.1) still holds. The integers $n$ such that $A_{n} \neq 0$ form an additive semigroup $S \subseteq \mathbb{N}$, and because $Q(A)_{1} \neq 0, S$ contains every sufficiently large integer.

For $n \in S$, we set $E_{n}=D\left(\bar{A}_{n}\right)$. If $A$ is generated in degrees $\leqq r$, the sequence $E_{n}$ has the property analogous to $(2.2 .2)$ :

$$
E_{n}=\bigcup_{1 \leqq r}\left(E_{i}+\sigma^{i} E_{n-1}\right)
$$

the sup being taken over those integers $i \leqq r$ such that $i$ and $n-i$ are in $S$. As in (2.4)(ii), this implies that

$$
E_{m}+\sigma^{-m} E_{n} \leqq E_{m+n}
$$

where $m, n \in S$. However, $E_{n}$ need not be positive.

The element $t \in Q(A)_{1}$ is arbitrary, and the effect of changing $t$ to $t^{\prime}=f t$, where $f \in K$, is to change $\bar{A}_{l}$ to $\bar{A}_{i} f_{l}^{-1}$, where $f_{i}=f f^{\sigma} \cdots f^{\sigma^{(t-1)}}$. So if $W$ is the divisor of $f$, then for $n \in S, E_{n}$ changes to

$$
E_{n}^{\prime}=E_{n}-\left(W+\sigma^{-1} W+\cdots+\sigma^{-(t-1)} W\right) .
$$

The next proposition, which generalizes Corollary 2.13 , will be used in Sections 5 and 6 to pass to Veronese subrings, and will allow us to eliminate the hypothesis that $R_{1} \neq 0$ is the main applications in those sections.

Proposition 2.21. Let $\sigma$ be an automorphism of infinite order of the function field $K$, and let $A$ be a finitely graded subring of $K[t ; \sigma]$ such that $Q(A)=$ $K\left[t, t^{-1} ; \sigma\right]$. Let $d$ be an integer such that $A_{d} \neq 0$, and let $R$ be the Veronese subring $A^{(d)}$. With notation as in (2.1), the divisor sequence $\left\{D_{n}=D_{n}^{R}\right\}$ for $R$ is a $\sigma^{d}$-divisor sequence.

Proof. Let $\left\{E_{n}, n \in S\right\}$ be the sequence of divisors associated to $A$ as above and let $\left\{F_{n}\right\}$ be the corresponding divisors associated to the finitely graded overring $A\langle t\rangle$. Then $\left\{F_{n}\right\}$ is a $\sigma$-divisor sequence with $F_{n} \geqq E_{n}$ for all $n$. It is now easy to prove that:

(i) $\left\{E_{n}\right\}$ is supported on finitely many orbits of $\sigma$.

(ii) On each infinite orbit $\mathscr{I}$ of $\sigma$, the multiplicities of the $E_{n}$ are bounded above. (Apply Proposition 2.11 to $\left\{F_{n}\right\}$.)

(iii) Let $p$ be a fixed point of $\sigma$ and write $\left.\left(E_{n}\right)\right|_{\{p\}}=s_{n} p$. Then there exists $c$ such that $s_{c r} / c r=\max _{n}\left\{s_{n} / n\right\}$, for all $r \geqq 1$. (Use the proof of Lemma 2.7.)

To obtain the sequence $\left\{D_{n}\right\}$, we choose an element $z \in A_{d}=R_{1}$, and write $R_{n}=\bar{R}_{n} z^{n}$. Then $D_{n}=D\left(\bar{R}_{n}\right)$. Say that $z=f t^{d}$, and $W=(f)$. By construction, the $D_{n}$ are non-negative divisors satisfying $D_{m}+\sigma^{-m} D_{n} \leqq D_{n+m}$, for all, $n, m \geqq 1$. Moreover, by $(2.20)$,

$$
D_{n}=E_{d n}-\left(W+\sigma^{-d} W+\cdots+\sigma^{-(i-1) d} W\right) .
$$


The properties (i)-(iii) given above carry over from $\left\{E_{n}\right\}$ to the Veronese sequence $\left\{E_{d n}\right\}$, and from there to the sequence $\left\{D_{n}\right\}$. In other words, neither (i) nor (ii) of Proposition 2.18 holds on any orbit. So $D_{n}$ is a $\sigma$-divisor sequence.

\section{The stable image of the curve}

Let $R$ be a finitely graded $k$-algebra, satisfying the hypotheses of (2.1). When $R$ is commutative and generated by $R_{1}$, then $R$ is determined, not by data on $X$, but by an invertible sheaf over a curve birational to $X$. A similar result holds in the noncommutative case, and the aim of this section is to find that curve. We fix the following notation:

Notation 3.1. Fix a graded $k$-algebra $R$ satisfying the hypotheses of (2.1), and such that the associated divisor sequence $\left\{D_{n}^{R}\right\}$ is a $\sigma$-divisor sequence. For each $n, \bar{R}_{n}$ defines a morphism $\phi_{n}: X \rightarrow \mathbb{P}\left(\bar{R}_{n}^{*}\right)$ [Ha, Theorem II.7.1]. We denote the image of $\phi_{n}$ by $Y_{n}$. For $m$ large $\bar{R}_{m}$ generates $K$ and so $Y_{n}$ is a curve birational to $X$ for all $n \gg 0$.

We will use the following elementary fact about rational maps:

Lemma 3.2. Let $\left\{V_{i}: 1 \leqq i \leqq 3\right\}$ be non-zero, finite dimensional $k$-subspaces of $K$ such that $V_{1} V_{2} \subseteq V_{3}$, and let $E_{i}=D\left(V_{i}\right)$ denote the associated divisors. Write $\theta_{i}: X \rightarrow \mathbb{P}\left(V_{i}^{*}\right)$ for the morphism defined by $V_{i}$ and set $Y_{i}=\theta_{i}(X)$. Assume that $Y_{3}$ is birational to $X$; thus, there is an induced rational map $\pi: Y_{3} \longrightarrow Y_{1}$ defined by $\pi=\theta_{1} \theta_{3}^{-1}$ on an appropriate open subset of $Y_{3}$. If $p \in X$ and if $E_{1}+E_{2}=E_{3}$ locally at $p$, then $\pi$ is defined at $q=\theta_{3}(p)$.

Proof. Pick $v \in V_{2}$ and $u_{0} \in V_{1}$ such that $E_{2}=-(v)$ and $E_{1}=-\left(u_{0}\right)$, locally at $p$. By hypothesis, $E_{3}=-\left(u_{0} v\right)$, locally at $p$. Pick a basis $\left\{u_{0}, \ldots, u_{r}\right\}$ of $V_{1}$ and a corresponding basis $\left\{u_{0} v, \ldots, u_{r} v, w_{r+1}, \ldots, w_{s}\right\}$ of $V_{3}$. These bases establish coordinates in $\mathbb{P}\left(V_{i}^{*}\right)$, for $i=1,3$, and define the maps $\theta_{1}$ and $\theta_{3}$; for example, $\theta_{1}$ is defined locally at $p$ by the coordinate functions $\left(u_{0} / u_{0}, \ldots, u_{r} / u_{0}\right)$.

Clearly, $\theta_{1}$ is also defined by the coordinate functions $\left(u_{0} v, \ldots, u_{r} v\right)$. Thus, the projection $\mathbb{P}\left(V_{3}^{*}\right)-\rightarrow \mathbb{P}\left(V_{1}^{*}\right)$ defined by projection onto the first $(r+1)$ coordinates induces the rational map $\pi=\theta_{1} \circ \theta_{3}^{-1}: Y_{3} \longrightarrow Y_{1}$. By construction, this is defined in a neighbourhood of $q=\theta_{3}(p)$.

Proposition 3.3. With notation as in (3.1), let $\mathscr{I}$ be a union of $\sigma$-orbits in $X$. Assume that there exists a divisor $Z$ and an integer $n_{0}$ such that $D_{n \mid s}=$ $Z+\sigma^{-1} Z+\cdots+\sigma^{-(n-1)} Z$ for all $n \geqq n_{0}$. Then:

(i) For $n \geqq n_{0}$ and $m \geqq 1$, the canonical rational map $\pi_{m, n}: Y_{m+n}--\rightarrow$ $Y_{n}$ induced by the maps $\phi_{i}$ is defined at every point of $\phi_{m}(\mathscr{I})$.

(ii) If $\mathscr{I}=X$, there is an integer $n_{1}$ such that $\pi_{m, n}$ is an isomorphism for all $n \geqq n_{1}$. Setting $Y:=Y_{n_{1}}$, this curve is birational to $X$, and $\sigma$ induces an automorphism of $Y$. (This automorphism will also be denoted by $\sigma$.) 
Remark. If $R$ is generated by $R_{1}$, then $\bar{R}_{n}=\bar{R}_{1} \bar{R}_{1}^{\sigma} \cdots \bar{R}_{1}^{\sigma^{n-1}}$. Hence $D_{n}=$ $D_{1}+\sigma^{-1} D_{1}+\cdots+\sigma^{-(n-1)} D_{1}$, and so the hypotheses of the corollary are satisfied, with $n_{1}=1$.

Proof. (i) Set $V_{1}=\bar{R}_{m}, V_{2}=\widetilde{R}_{n}^{\sigma^{\prime \prime \prime}}$ and $V_{3}=\bar{R}_{m+n}$. By hypothesis, the associated divisors $E_{1}=D_{m \mid \cdot 9}, E_{2}=\left.\sigma^{-m}\left(D_{n}\right)\right|_{,}$and $E_{3}=\left.D_{m+n}\right|_{9}$ satisfy $E_{1}+E_{2}=E_{3}$. Thus Lemma 3.2 applies, and it shows that $\pi_{m, n}$ is defined on $\phi_{m}(\mathscr{I})$.

(ii) For $n \gg n_{0}, K$ is generated by $\bar{R}_{n}$ and so $X$ is birational to $Y_{n}$. By part (i) $\pi_{n ! n}$ is defined and, by construction, it is surjective. Since (i) $X$ is the integral closure of ${ }^{O}{ }_{\gamma}$, the set of integral schemes lying between $X$ and $Y_{n}$ satisfies ACC. Therefore, $\pi_{1, n}: Y_{n+1} \rightarrow Y_{n}$ is an isomorphism for all large $n$ and so $n_{1}$ exists.

Let $n \geqq n_{1}$. By convention, $f^{\sigma}(x)=f(\sigma(x))$, for $x \in X$ and $f \in K$. Thus, with suitable choice of coordinates in the projective space $\mathbb{P}\left(\bar{R}_{n}^{*}\right)$, the morphism defined by $\bar{R}_{n}^{\bar{\sigma}^{m}}$ is $\phi_{n} \circ \sigma^{m}$, and its image is $Y_{n}=Y$. Since $D_{m}+\sigma^{-m} D_{n}=D_{m+n}$, Lemma 3.2 produces a morphism $\pi_{m}^{\prime}: Y_{n+m} \rightarrow Y_{n}$, such that $\pi_{m}^{\prime} \circ \phi_{n+m}=$ $\phi_{n} \circ \sigma^{m}$. Thus, if $\tau_{m}=\pi_{m}^{\prime} \circ \pi_{n+m, n}^{-1}$, then $\tau_{m} \circ \phi_{n}=\phi_{n} \circ \sigma^{m}$. It follows that $\tau=\tau_{m-1} \tau_{m}^{-1}$ is the required automorphism.

When $R$ is not generated by $R_{1}, \pi_{n}$ need not be defined everywhere and so more care will be needed. For example, let $X=\mathbb{P}^{\mathrm{l}}$, with $k\left(\mathbb{P}^{\mathbf{l}}\right)=k(u)$ and let $R=k\left\langle z, u z, u^{4} z^{2}\right\rangle \subset k(u)[z ; \sigma]$, where $\sigma(u)=\lambda u$ for any $\lambda \in k^{*}$ (including $\lambda=1$ ). Then, $\bar{R}_{n}$ has basis $\left\{1, u, \ldots, u^{2 n-1}\right\}$ when $n$ is odd but $\left\{1, u, \ldots, u^{2 n-2}, u^{2 n}\right\}$ when $n$ is even. Consequently, if $p$ is the pole of $u$, then $D_{n}=(2 n-1) p$ if $n$ is odd but $D_{n}=(2 n) p$ if $n$ is even. It follows that, for all $m, Y_{2 m+1} \cong X$ but $Y_{2 m}$ has a cusp at infinity. In this example, as is true for any commutative Noetherian graded $k$-algebra, one can obtain a ring generated in degree one by replacing $R$ by a suitably large Veronese ring. Thus, Proposition 3.3 may be applied to that Veronese ring. However, as was remarked in the last section, there exist finitely graded algebras none of whose Veronese rings are generated in degree one. Nevertheless, when $|\sigma|=\infty$, the conclusion of Proposition 3.3 still holds (see Proposition 3.5).

Lemma 3.4. With the notation of (3.1), assume that $|\sigma|=\infty$ and let $\mathscr{I}$ be an infinite orbit of $\sigma$. Then, for $n \gg 0$, the curve $Y_{n}$ is smooth at every point of $\mathscr{I}$.

Proof: Throughout this proof, we assume that $i, j \gg 0$ and we set $n=i+j$. Let $\Omega_{\mathscr{g}}$ denote the gap divisor on $\mathscr{I}$, as defined after Corollary 2.12, and let $T$ denote the support of $\Omega_{\mathscr{g}}$ on $X$. Set $E_{n}=D_{n \mid \mathscr{y}}$. By $(2.12 .1), E_{i}+\sigma^{-i} E_{j}+$ $\sigma^{-i} \Omega_{q}=E_{n}$. Thus, by Lemma 3.2 with $V_{1}=\bar{R}_{i}, V_{2}=\bar{R}_{j}^{\sigma^{i}}$ and $V_{3}=\bar{R}_{n}$, the rational map $\pi: Y_{n} \rightarrow-\rightarrow Y_{t}$ is defined everywhere on $\phi_{n}(\mathscr{I})$, except possibly on $\phi_{n}\left(\sigma^{-i} T\right)$. Let $S_{i}$ denote the points $p \in \mathscr{I}$ such that $\phi_{i}(p)$ is a singular point of $Y_{i}$. If $y$ is a singular point of $Y_{n}$ and if $\pi$ is defined at $y$, then $\pi(y)$ is a singular point of $Y_{i}$. Therefore $S_{n} \subseteq S_{i} \cup \sigma^{-i} T$. 
We repeat this argument with $V_{1}=\bar{R}_{j}^{\sigma^{t}}$ and $V_{2}=\bar{R}_{t}$. As in the proof of Proposition 3.3(ii), the morphism $\theta: X \rightarrow \mathbb{P}\left(V_{1}^{*}\right)$ defined by $V_{1}$ is $\theta=\phi, \circ \sigma^{l}$. Therefore the set of points $p \in X$ such that $\theta(p)$ is singular is $\sigma^{-i} S_{j}$, and the argument of the last paragraph implies that $S_{n} \subseteq \sigma^{-i} S_{j} \cup \sigma^{-i} T$. Interchanging the rôles of $i$ and $j$ shows that $S_{n} \subseteq \sigma^{-\prime} S_{1} \cup \sigma^{-1} T$ and hence that $S_{n} \subseteq\left(S_{l} \cup \sigma^{-i} T\right) \cap\left(\sigma^{-j} S_{i} \cup \sigma^{-\prime} T\right)$. This intersection is empty if $j \gg i$.

Proposition 3.5. Assume that (2.15) holds for the graded ring $R$. Then, for $n \gg 0$ and $m \geqq 1$, the canonical rational map $\pi_{m, n}: Y_{m+n} \longrightarrow Y_{n}$ induced by the maps $\phi_{i}$ is defined at every point of $Y_{m+n}$, and it is an isomorphism. If $Y:=Y_{n}$, then $\sigma$ induces an automorphsim, again written $\sigma$, on $Y$.

Proof: For $n \gg 0$, the previous lemma implies that $Y_{n+m}$ is smooth on each infinite orbit of $\sigma$. Thus, $\pi_{m, n}$ is certainly defined on these orbits [Ha, Proposition I.6.8]. By (2.15) and Proposition 3.3(i), $\pi_{m, n}$ is defined at the fixed points of $\sigma$ and so it is defined on all of $Y_{m+n}$. The argument used in the proof of Proposition 3.3 shows that $\pi_{m, n}$ is an isomorphism for $n \gg 0$ and that $\sigma$ induces an automorphism of $Y_{n}$.

Remark. In applications of Propositions 3.3 and 3.5, we will use $\phi: X \rightarrow Y$ and $\pi_{t}: Y \rightarrow Y_{i}$ to denote the induced morphisms.

We now wish to derive relations for coherent sheaves on $Y$ analogous to the formulae of Lemma 2.17 for divisors on $X$. We work as much as possible with coherent sheaves $\mathscr{M}$ on $Y$ which are fractional ideals, that is, coherent subsheaves of the constant sheaf $K$ on $Y$. The notation $\mathscr{U} \cdot \mathscr{N}$ will denote the product ideal in $K$. If either $\mathscr{M}$ or $\mathscr{N}$ is an invertible sheaf, then this product ideal is canonically isomorphic to the tensor product $\mathscr{M} \otimes \mathscr{N}$, and we will use the tensor product notation when applicable. (The unadorned $\otimes$ stands for $\left.Q_{C_{Y}}.\right)$ If $\mathscr{M}$ is invertible, $\mathscr{M}^{\vee}$ will denote the dual sheaf $\operatorname{Hom}\left(\mathscr{M}, \mathscr{O}_{Y}\right)$. If $D$ is a divisor supported on the smooth points of $Y$, then $\mathcal{O}_{Y}(D)$ is an invertible sheaf, and $\mathscr{M}(D)$ will denote the product $\mathscr{M} \otimes \mathscr{O}_{Y}(D)$.

Lemma 3.6. Let $\phi: X \rightarrow Y$ be a surjective morphism of Noetherian schemes, and let $\mathscr{N} \subseteq \mathscr{M}$ be invertible sheaves on $Y$. Let $\mathscr{N}^{\prime}=\mathscr{N} \cdot \mathscr{O}_{X}$ and $\mathscr{M}^{\prime}=\mathscr{M} \cdot \mathscr{O}_{X}$ denote the induced invertible sheaves on $X$. If $\cdot \mathscr{N}^{\prime}=\mathscr{M}^{\prime}$, then $\mathscr{N}=\mathscr{M}$.

Proof. This assertion may be verified locally, so we may assume that $\mathscr{M}=\mathfrak{O}_{Y}$ and that $\mathscr{N}$ is an ideal of $\mathcal{O}_{Y}$, say the ideal of the closed subscheme $Z$. Then $\mathscr{M}^{\prime}=\mathscr{O}_{X}$ and $\mathscr{N}^{\prime}$ is the ideal sheaf of the inverse image $\phi^{-1}(Z)$. The assertion $\mathscr{N}^{\prime}=\mathscr{M}^{\prime}$ translates to $\phi^{-1}(Z)=\emptyset$. Since $\phi$ is surjective, $\phi^{-1}(Z)=\emptyset$ implies $Z=\emptyset$.

We will frequently wish to use the following notation when dealing with automorphisms of infinite order. 
Notation 3.7. Let $R$ be a ring satisfying (2.15), and fix an integer $n_{1}$ such that: (i) Equality holds in (2.11)(ii) for all $n \geqq n_{1}$ and all infinite orbits $\mathscr{F}$ of $\sigma$.

(ii) For each fixed point $p$ of $\sigma$ on $X$, the multiplicity of $D_{n}$ on $p$ equals $s_{p} n$, for all $n \geqq n_{1}$.

(iii) The maps $\phi: X \rightarrow Y_{n}$ are defined, and $Y_{n} \cong Y$ for all $n \geqq n_{1}$. For $i \geqq 1$, set $\mathscr{R}_{i}=\bar{R}_{i} \cup_{Y}$. If $i \geqq n_{1}$, then $\bar{R}_{i}$ defines an embedding $\pi_{i}$ : $Y \rightarrow \mathbb{P}=\mathbb{P}\left(\bar{R}_{i}^{*}\right)$ and so $\left[\mathrm{Ha}\right.$, Theorem II,7.1] implies that $\mathscr{R}_{i}=\pi_{i}^{*} \mathbb{C}_{\mathbb{P}}(1)$ is invertible. (For $i<n_{1}, \mathscr{n}_{l}$ need not be invertible.) By Lemma 3.4, $Y$ is smooth on every point of the support of the gap divisor $\Omega$, and so we may regard $\Omega$ as a Cartier divisor on $Y$. Thus, $C_{Y}(\Omega)$ is an invertible sheaf. For

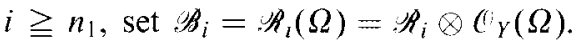

Lemma 3.8. Keep the notation of (3.7). Then:

(i) $\mathscr{R}_{i} \cdot \mathscr{R}_{j}^{\sigma^{l}} \subseteq \mathscr{R}_{i+j}$ for all $i, j \geqq 1$, and hence $\mathscr{B}_{i} \otimes \cdot \mathscr{R}^{\sigma^{\prime}} \subseteq \mathscr{B}_{1+j}$ for all $i \geqq n_{1}$ and $j \geqq 1$.

(ii) $\mathscr{h}_{i}\left(\sigma^{-i} \Omega\right) \otimes \mathscr{h}_{j}^{\sigma^{\prime}}=\mathscr{h}_{1+}$ for $i, j \geqq n_{1}$.

(iii) $\mathscr{R}_{1} \otimes \mathscr{B}_{j}^{\sigma^{t}}=\mathscr{R}_{i+1}$ for $i, j \geqq n_{1}$.

(iv) $\mathscr{B}_{l} \otimes \mathscr{B}_{1}^{\sigma^{l}}=\mathscr{B}_{l+j}$ for $i, j \geqq n_{1}$.

Proof. (i) follows directly from (2.1.1) and the definitions. To check (ii)-(iv), we may work locally at each point of $Y$. Away from the fixed points, $Y$ is smooth, and so the assertions follow from the corresponding formulae (2.16) and (2.17)(i), (ii) for divisors on $X$. Locally at the fixed points, $\sigma^{-1} \Omega$ is zero for any $j$. Hence $\mathscr{R}_{t}=\mathscr{h}_{i}\left(\sigma^{-l} \Omega\right)=\mathscr{B}_{i}$ locally at such a point, and (ii)-(iv) will follow if we prove that

$$
\mathscr{R} \otimes \mathscr{R}_{j}^{\sigma^{\prime}}=\mathscr{R}_{i+j} \text { locally at a fixed point }
$$

Set $: \mathscr{R}_{i}^{\prime}=\mathscr{R}_{1} \cdot \mathscr{O}_{X}$ and note that $: \mathscr{R}_{i}^{\prime}=\bar{R}_{I} C_{X}=\mathcal{O}_{X}\left(D_{i}\right)$. Then $(2.14)$ and (3.7)(ii) imply that $\mathscr{R}_{i}^{\prime} \otimes\left(\mathscr{R}_{i}^{\prime}\right)^{\sigma^{\prime}}=\mathscr{R}_{i+j}^{\prime}$ at each fixed point. Thus (3.8.1) follows from part (i) and Lemma 3.6. This completes the proof.

\section{Domains of dimension two which are generated in degree one}

Let $R$ be an algebra satisfying the hypotheses of (2.1) and generated in degree one, and define $Y$ and its automorphism $\sigma$ as in the last section. The aim of this section is to prove that $R$ has finite codimension in the twisted homogeneous coordinate ring $B(Y, \mathscr{L}, \sigma)$, as defined below (see Theorem 4.7). The significance of this result is that the properties of twisted homogeneous coordinate rings are rather pleasant; many of them are the same as for the commutative ring $B(Y, \mathscr{L}, i d)$ (see Corollary 4.8 for the details). 
For any triple $(Y, \mathscr{L}, \sigma)$ consisting of a projective scheme $Y$, an automorphism $\sigma$ of $Y$, and an invertible sheaf $\mathscr{L}$ on $Y$, the twisted homogeneous coordinate ring $B=B(Y, \mathscr{L}, \sigma)$ is defined as follows: $B$ is the graded $k$-algebra $\bigoplus_{i \geqq 0} B_{n}$, where $B_{n}=\bar{B}_{n} z^{n}$, for $\bar{B}_{n}=H^{0}\left(Y, \mathscr{L}_{n}\right)$ and $\mathscr{L}_{n}=\mathscr{L} \otimes \mathscr{L}^{\sigma} \otimes \cdots \otimes \mathscr{L}^{\sigma^{n-1}}$. The multiplication map $\bar{B}_{m} \otimes \bar{B}_{n}^{\sigma^{m}} \rightarrow \bar{B}_{m+n}$ is defined by taking global sections of the natural isomorphism $\mathscr{L}_{m} \otimes \mathscr{L}_{n}^{\sigma^{m}} \rightarrow \mathscr{L}_{m+n}$. (Here $z$ can be thought of as a dummy variable which encodes the automorphism $\sigma$. It has been introduced for consistency with the earlier parts of this paper.)

We recall some results of $[\mathrm{AV}]$ about the structure of $B(Y, \mathscr{L}, \sigma)$. An invertible sheaf $\mathscr{L}$ is called $\sigma$-ample if, for every coherent sheaf $\mathscr{F}$, one has $H^{q}\left(Y, \mathscr{F} \otimes \mathscr{L}_{n}\right)=0$, for all $q>0$ and $n \gg 0$. Given a graded ring $B$, let gr- $B$ denote the category of Noetherian graded right $B$-modules and let $\tau(B)$ denote the full subcategory of graded, finite dimensional $B$-modules. Write $\overline{g r}$ $B=(\operatorname{gr}-B) / \tau(B)$ for the quotient category. Two graded right $B$-modules $M$ and $M^{\prime}$ are called equivalent if their images in $\overline{\mathrm{gr}}-B$ are equal. If $B$ is Noetherian, then two finitely generated, graded modules $M, M^{\prime}$ are equivalent if and only if their tails $M_{\geqq n}=\bigoplus_{i \geqq n} M_{i}$ and $M_{\geqq n}^{\prime}$ are equal for sufficiently large $n$. Denote by ${ }^{\prime}{ }_{Y}$-mod the category of coherent sheaves on a scheme $Y$.

Let $(Y, \mathscr{L}, \sigma)$ be a triple as above, assume that $\mathscr{L}$ is both $\sigma$-ample and $\sigma^{-1}$ ample, and let $B=B(Y, \mathscr{L}, \sigma)$. By [AV, Theorem 1.3], $B$ is Noetherian ${ }^{1}$, and there is an equivalence of categories $O_{Y}-\bmod \rightarrow \overline{\mathrm{gr}}-B$, under which a coherent sheaf $\mathscr{N}^{\circ}$ is mapped to the module $\Gamma_{*}(\mathscr{N})=\bigoplus_{i \in \mathbb{Z}} H^{0}\left(\mathscr{N}^{\prime} \otimes \mathscr{L}_{n}\right)$.

The following result complements the results of [AV] and gives a noncommutative analogue of a theorem of Serre.

Theorem 4.1. Let $\sigma$ be an automorphism of a projective scheme $Y$, and let $\mathscr{L}$ be an invertible sheaf on $Y$ which is both $\sigma$-ample and $\sigma^{-1}$-ample. Set $B=B(Y, \mathscr{L}, \sigma)$, as above. Let $R=\bigoplus_{i \geqq 0} \bar{R}_{n} z^{n} \subseteq B$ be a graded subring of subexponential growth. Assume that, for $n \gg 0, \bar{R}_{n}$ generates $\mathscr{L} \otimes \mathscr{L}^{\sigma} \otimes \cdots \otimes$ $\mathscr{L}^{\sigma^{n-1}}$ and that the map $Y \rightarrow \mathbb{P}\left(\bar{R}_{n}^{*}\right)$ defined by $\bar{R}_{n}$ is an embedding. Then $R_{m}=B_{m}$ for all $m \gg 0$.

We conjecture that the growth condition follows from the other hypotheses. Note also that $R$ is not assumed to be finitely generated in Theorem 4.1. However, this follows from the conclusion of the theorem. Indeed, by [AV], $B$ is a finitely generated, Noetherian algebra. Since $B / R$ is finite dimensional, Lemma 1.14 implies that $R$ is Noetherian and finitely generated.

The proof of Theorem 4.1 will be obtained through a series of lemmas. The first is a standard application of $\sigma$-ample sheaves that will be used frequently.

Lemma 4.2. Let $\mathscr{F}$ be a coherent sheaf and $\mathscr{L}$ an invertible sheaf on a projective scheme $Y$ such that $\mathscr{L}$ is both $\sigma$-ample and $\sigma^{-1}$-ample. Then:

${ }^{1}$ In [AV], this theorem carelessly states that $B$ is Noetherian if $\mathscr{L}$ is $\sigma$-ample. What is proved is that, with our conventions, $B$ is right Noetherian. 
(i) For $n \gg 0$, the sheaf $\widetilde{F} \otimes \mathscr{L}_{n}$ is generated by its sections.

(ii) Let $V$ be a vector space of glohal sections of a coherent sheaf $\mathscr{F}$ which generates $\bar{F}$, and let $r \in \mathbb{N}$. For $m \gg 0$, the natural map $V \otimes H^{0}\left(Y, \mathscr{L}_{m}^{\sigma^{\prime \prime}}\right) \rightarrow H^{0}\left(Y, \mathscr{F} \otimes \mathscr{L}_{m}^{\sigma^{\prime}}\right)$ is surjective.

(iii) Keep the hypotheses of part (ii). Then, for all $m \gg 0$, the natural homomorphism $V^{\sigma^{\prime \prime}} \otimes H^{0}\left(Y, \mathscr{L}_{m}\right) \rightarrow H^{0}\left(Y, \widetilde{\mathscr{F}}^{m} \otimes \mathscr{L}_{m}\right)$ is surjective.

Proof. (i) This is [AV, Proposition 3.2].

(ii) It follows easily from the definition that $\mathscr{L}$ is $\sigma$-ample if and only if $\mathscr{L}^{\sigma^{r}}$ is $\sigma$-ample and so we may assume that $r=0$.

There is a short exact sequence

$$
0 \rightarrow \mathscr{H} \rightarrow V \otimes_{k}{ }^{(}{ }_{Y} \rightarrow \mathscr{F} \rightarrow 0 .
$$

Since $\mathscr{L}$ is $\sigma$-ample, $H^{1}\left(Y, \mathscr{H} \otimes \mathscr{L}_{m}\right)=0$, for all $m \gg 0$. Thus, tensoring this exact sequence with $\mathscr{L}_{m}$ and taking global sections gives the exact sequence

$$
0 \rightarrow H^{0}\left(Y, \mathscr{H} \otimes \mathscr{L}_{m}\right) \rightarrow V \otimes_{k} H^{0}\left(Y, \mathscr{L}_{m}\right) \rightarrow H^{0}\left(Y, \mathscr{F} \otimes \mathscr{L}_{m}\right) \rightarrow 0,
$$

as required.

(iii) In this case, applying $\sigma^{-m}$ to the given map, it suffices to prove that the map $V \otimes H^{0}\left(Y, \mathscr{L}_{m}^{\sigma^{-m}}\right) \rightarrow H^{0}\left(Y, \mathscr{F} \otimes \mathscr{L}_{m}^{\sigma^{-m}}\right)$ is surjective. Now, $\mathscr{L}_{m}^{\sigma^{-m}}=$ $\mathscr{L}^{\sigma^{-1}} \otimes \cdots \otimes \mathscr{L}^{\sigma^{-m}}$. Thus, this result follows from part (ii), with $\sigma$ replaced by $\sigma^{-1}$ and $r=1$.

Lemma 4.3. It suffices to prove Theorem 4.1 for some pair of Veronese rings $R^{(d)} \subseteq B^{(d)}$.

Proof. Observe that the hypotheses of Theorem 4.1 do carry over to these Veronese rings. If $R_{r}^{(d)}=B_{r}^{(d)}$ for $r \gg 0$, then $\bar{R}_{r d}=\bar{B}_{r d}$ for all $r \gg 0$. Let $V=\bar{R}_{\imath}$ and $\mathscr{F}=\mathscr{L}_{i}$ for $i \gg 0$. Then Lemma 4.2(ii), with $m=r d$ implies that the multiplication map

$$
\bar{R}_{i} \otimes \bar{R}_{r d}^{\sigma^{d}}=\bar{R}_{i} \otimes \bar{B}_{r d}^{\sigma^{\prime}} \rightarrow \bar{B}_{1+r d}
$$

is surjective for $r \gg 0$. Hence, $\bar{R}_{i+r d}=\bar{B}_{l+r d}$, as required.

Lemma 4.4. Let $B=B(Y, \mathscr{L}, \sigma)$ be as above. The functor $\Gamma_{*}$ defines a bijection between the set of equivalence classes of two-sided ideals $M$ of $B$ and the set of $\sigma$-invariant closed subschemes $Z$ of $Y$.

Proof. This is an elementary computation. Let $\mathscr{J}$ denote the ideal sheaf of a subscheme $Z$ and set $M=\Gamma_{*}(\mathscr{J})_{\geq 0}$ and $M^{(m)}=\Gamma_{*}\left(\mathscr{J}^{m}\right)_{\geq 0}$. Applying the functor $\Gamma_{* \geqq 0}$ to the inclusion $0 \rightarrow \mathscr{J} \rightarrow \mathcal{O}_{Y}$, yields an inclusion of right $B$-modules $0 \rightarrow M \rightarrow B$. Here, the inclusion $M_{n}=H^{0}\left(Y, \mathscr{J} \otimes \mathscr{L}_{n}\right) \subseteq B_{n}=$ $H^{0}\left(Y, \mathscr{L}_{n}\right)$ is the map obtained by taking global sections of the multiplication map $\mathscr{J} \otimes \mathscr{L}_{n} \rightarrow \mathscr{C}_{Y} \otimes \mathscr{L}_{n}=\mathscr{L}_{n}:$

$$
\alpha \otimes \lambda_{0} \otimes \cdots \otimes \lambda_{n-1}^{\sigma^{n-1}} \mapsto \alpha \lambda_{0} \otimes \cdots \otimes \lambda_{n-1}^{\sigma^{n-1}} .
$$


So left multiplication by $B_{m}$ on such a section is given by

$$
\begin{aligned}
& \left(\mu_{0} \otimes \cdots \otimes \mu_{m-1}\right) \cdot\left(\alpha \lambda_{0} \otimes \cdots \otimes \lambda_{n-1}^{\sigma^{n-1}}\right) \\
& \quad=\left(\mu_{0} \otimes \cdots \otimes \mu_{m-1}\right) \otimes\left(\alpha \lambda_{0} \otimes \cdots \otimes \lambda_{n-1}^{\sigma^{n-1}}\right)^{\sigma^{n}} \\
& \quad=\alpha^{\sigma^{m}}\left(\mu_{0} \otimes \cdots \otimes \mu_{m-1}\right) \otimes\left(\lambda_{0} \otimes \cdots \otimes \lambda_{n-1}^{\sigma^{n-1}}\right)^{\sigma^{m}}
\end{aligned}
$$

which yields an element of $\mathscr{J}^{\sigma^{m}} \otimes \mathscr{L}_{m+n}$. Thus, if $b \in B_{m}$ and $a \in M_{n}$, then $b a \in M_{m+n}^{(m)}$. If $Z$ is a $\sigma$-invariant closed subscheme of $Y$, then $M^{(m)} \subseteq M$, and $M$ is an ideal of $B$.

Conversely, suppose that $M=\Gamma_{*}(\mathscr{J})_{\geqq 0}$ is a two-sided ideal of $B$. If $m, n$ are sufficiently large then, because $\mathscr{L}$ is $\sigma$-ample, $\mathscr{L}_{m}$ and $\mathscr{J} \otimes \mathscr{L}_{n}$ are generated by their global sections $B_{m}$, respectively $M_{n}$. Since $B_{m} M_{n} \subseteq M_{m+n}$, it follows that $\mathscr{J}^{\sigma^{m}} \otimes \mathscr{L}_{m+n} \subseteq \mathscr{J} \otimes \mathscr{L}_{m+n}$. Hence $\mathscr{J}^{\sigma^{m}} \subseteq \mathscr{J}$ and so $\mathscr{J}^{\sigma^{m}}=\mathscr{I}$. Since this is true for all large $m, \mathscr{J}^{\sigma}=\mathscr{J}$. Thus, $\mathscr{J}$ is the ideal sheaf of a $\sigma$-invariant subscheme $Z$.

Lemma 4.5. $B$ is finitely generated as a left and right $R$-module.

Proof. Fix $n \gg 0$ for which $\bar{R}_{n}$ generates $\mathscr{L}_{n}$. Then, Lemma 4.2 (ii) implies that the multiplication map

$$
\bar{R}_{n} \otimes \bar{B}_{m}^{\sigma^{n}}=\bar{R}_{n} \otimes H^{0}\left(Y, \mathscr{L}_{m}^{\sigma^{n}}\right) \rightarrow H^{0}\left(Y, \mathscr{L}_{n+m}\right)=\bar{B}_{n+m}
$$

is surjective. Hence, $B$ is a finitely generated left $R$-module. An analogous argument, using Lemma 4.2 (iii), shows that $B_{R}$ is finitely generated.

Proof of Theorem 4.1. Let $Z$ be a $\sigma$-invariant closed subscheme of $Y$. We can identify $\Gamma_{*}\left(\mathscr{O}_{Z}\right)_{\geqq 0}$ as the ring $B_{Z}=B\left(Z,\left.\mathscr{L}\right|_{Z},\left.\sigma\right|_{Z}\right)$. Applying the functor $\Gamma_{* \geqq 0}$ to the exact sequence $0 \rightarrow \mathscr{J} \rightarrow \mathcal{O}_{Y} \rightarrow \mathfrak{O}_{Z} \rightarrow 0$, yields an exact sequence of right $B$-modules

$$
0 \rightarrow M \rightarrow B \rightarrow B_{Z} \rightarrow \varepsilon \rightarrow 0,
$$

where $\varepsilon$ is finite dimensional [AV, Lemma 3.13]. Thus, if $B^{\prime}$ denotes the image of $B$ in $B_{Z}$, then $B_{Z} / B^{\prime}$ is finite dimensional. Let $R^{\prime}=(R+M) / M$. The hypotheses of the theorem carry over to the pair $R^{\prime} \subseteq B_{Z}$; in particular, the map $Z \rightarrow \mathbb{P}\left(V^{*}\right)$, defined by $V=\left(\widetilde{R}_{n}+M\right) / M$, is the restriction of the map $Y \rightarrow \mathbb{P}\left(\vec{R}_{n}^{*}\right)$, hence it is an embedding of $Z$ if $n$ is large. By Noetherian induction on $Y$, we may assume that $R$ does not have finite codimension in $B$, but that $R^{\prime}$ has finite codimension in $B_{Z}$ for every proper $\sigma$-invariant subscheme $Z \subseteq Y$, or equivalently, that for every non-zero ideal $M$ of $B$, the ring $(R+M / M)$ has finite codimension in $B / M$. This implies that $R$ and $B$ have no ideals in common. Observe that, as the induction is on $Y$, Lemma 4.3 implies that $R^{(d)}$ and $B^{(d)}$ also have no ideals in common.

The next lemma will allow us to reduce the proof to the case that $Y$ is an irreducible variety. 
Lemma 4.6. Suppose, in addition to the above assumptions, that there exist non-zero, $\sigma$-invariant, ideal sheaves $\mathscr{I}_{1}, \ldots, \mathscr{I}_{1}$ of $C_{Y}$ such that $\mathscr{I}_{1} \mathscr{I}_{2} \ldots \mathscr{I}_{1}=$ 0 . Then Theorem 4.1 holds for $Y$.

Proof. By induction, we may assume that $\ell=2$. Let $M_{r}=\Gamma_{*}\left(\mathscr{I}_{i}\right)_{\geqslant 0}$, and we write $Z_{l}$ for the closed subscheme defined by $\mathscr{I}_{i}$. Since $\mathscr{I}_{1}$ is a (right and left) $\mathcal{O}_{Z_{2}}$-module, the right action of $B$ on $M_{1}$ factors through the quotient $B_{2}=B / M_{2} \subseteq B_{Z_{2}}$. Thus, $M_{1}$ is a right (and, analogously, left) $B_{2}$-module. Since $B$ is Noetherian, this implies that $M_{1}$ is a finitely generated left or right $B_{2}$-module. Write $R_{2}=\left(R+M_{2}\right) / M_{2} \subseteq B_{2}$. By the inductive hypothesis, $B_{2} / R_{2}$ is finite dimensional and so $R_{2}$ is Noetherian. Thus, $M_{1}$ and $N_{1}=R \cap M_{1}$ are Noetherian (left and right) $R_{2}$-modules. This implies that $N_{1}^{\prime}=B_{2} N_{1} B_{2}$ is a Noetherian $R_{2}$-module and that $N_{1}^{\prime} / N_{1}$ is finite dimensional. Consequently, for $m \gg 0,\left(N_{1}\right)_{\geqq m}=\left(N_{1}^{\prime}\right)_{\geqq m}$ is a $B_{2}$-module and hence an ideal of $B$. However, by the inductive hypothesis, $R$ and $B$ have no ideals in common; thus $\left(N_{1}\right)_{\geq m}=0$.

This implies that $R \hookrightarrow B /\left(M_{1}\right)_{\geqq m}$. We may replace $R$ by $k+R_{\geqq m}$ without loss and so conclude that $R \hookrightarrow B / M_{1}=B\left(Z_{1},\left.\mathscr{L}\right|_{Z_{1}},\left.\sigma\right|_{Z_{1}}\right)$. Then for every $n$, the map $\phi_{n}: Y \rightarrow \mathbb{P}\left(\bar{R}_{n}^{*}\right)$ defined by $\bar{R}_{n}^{*}$ factors through $Z_{1}$. This contradicts the hypothesis that $\phi_{n}$ is an embedding for $n \gg 0$.

Completion of the proof of Theorem 4.1. The nilradical $\mathcal{N}$ of ${ }^{\theta_{Y}}$ is $\sigma$ invariant. So if $\mathscr{N} \neq 0$, then there is a nonzero invariant ideal $\mathscr{I}$ such that $\mathscr{J}^{2}=0$. This contradicts Lemma 4.6 and implies that $Y$ is reduced. Suppose that $Y$ is reducible. By Lemma 4.3, we may freely replace $R$ and $B$ by their Veronese rings $R^{(d)} \subseteq B^{(d)}$ and this has the effect of replacing $\sigma$ by $\sigma^{d}$. Now, $\sigma$ operates on the set of irreducible components of $Y$, and passing to the appropriate Veronese rings, we may assume that this operation is trivial. Thus, each irreducible component $Y_{l}$ of $Y$ corresponds to a $\sigma$-invariant ideal sheaf $\mathscr{I}_{i}$ of $\mathscr{O}_{Y}$ and $\prod \mathscr{I}_{i}=0$. Once again, this contradicts Lemma 4.6.

Thus $Y$ is reduced and irreducible. Therefore $B$ and $R$ are domains and, since it has subexponential growth, $R$ is an Ore domain, and we may form the graded quotient ring $Q(R)$ of $R$. By Lemma 4.3, again, we may replace $R$ by a Veronese ring and assume that $\bar{R}_{1} \neq 0$. By hypothesis, the map to projective space defined by $\bar{R}_{n}$ is an embedding of $Y$ for some $n$. Therefore, the subfield of $Q(R)$ generated by $\bar{R}_{n}$ is the field of fractions $K$ of $Y$, which is also the degree-zero part of $Q(B)$. Since $R$ contains an element $z$ of degree one, $Q(R)=Q(B)=K\left[z, z^{-1} ; \sigma\right]$. In particular, $B / R$ is a torsion (left or right) $R$-module.

Since $B / R$ is a finitely generated, torsion right $R$-module, the left annihilator $U=\ell$ - $\operatorname{ann}_{R}(B / R)$ is a non-zero ideal of $R$. Similarly, $V=r$-ann $R(B / R)$ and $V U$ are non-zero ideals of $R$. Since $V U$ is an ideal of $B$, this contradicts our inductive assumption and completes the proof of the theorem.

We now return to rings of Gelfand-Kirillov dimension two and apply Theorem 4.1. 
Theorem 4.7. Let $R=\bigoplus_{i \geqq 0} R_{i}$ be a connected graded domain, generated by $R_{1}$ as a $k$-algebra, with $G K \operatorname{dim}(R)=2$ and graded quotient ring $Q(R) \cong K\left[z, z^{-1} ; \sigma\right]$. Then, there exist a projective curve $Y$ birational to $X$, an automorphism $\sigma$ of $Y$ and an ample, invertible sheaf $\mathscr{L}$ over $Y$ such that $R \subseteq B=B(Y, \mathscr{L}, \sigma)$, with $B / R$ a finite dimensional $k$-vector space.

Proof. This follows from Theorems 1.1 and 4.1 combined with Proposition 3.3. In more detail, by Theorem 1.1, $R$ satisfies the hypotheses of (2.1) and hence satisfies the hypotheses of Proposition 3.3 (see the remark after that corollary). Let $Y$ be the curve defined by Proposition 3.3, with its induced action of $\sigma$, and set $\mathscr{L}=\pi_{1}^{*} \mathscr{O}_{\mathbb{P}_{1}}(1)$, where $\pi_{n}: Y \rightarrow Y_{n} \subseteq \mathbb{P}_{n}=\mathbb{P}\left(\bar{R}_{n}^{*}\right)$ is the map defined by $\bar{R}_{n}$. By construction, $\mathscr{L}$ is generated by the sections $\bar{R}_{1}$ and hence $\mathscr{L}_{n}=\mathscr{L} \otimes \cdots \otimes \mathscr{L}^{\sigma^{n-1}}$ is generated by the sections $\bar{R}_{1} \cdots \bar{R}_{1}^{\sigma^{n-1}}=\bar{R}_{n}$, for all $n \geqq 1$. Since $Y$ is a curve and $\mathscr{L}$ is ample, [AV, Corollary 1.6] implies that $\mathscr{L}$ is both $\sigma$-ample and $\sigma^{-1}$-ample. Finally, for all $n \gg 0, \pi_{n}$ is an embedding by Proposition 3.3. Thus, the hypotheses of Theorem 4.1 are indeed satisfied for $R \subseteq B(Y, \mathscr{L}, \sigma)$.

The strength of Theorem 4.7 is that it allows one to use the ring $B=$ $B(Y, \mathscr{L}, \sigma)$ to give a detailed description of the structure of $R$, as the next result illustrates.

Corollary 4.8. Let $R$ be a ring satisfying the hypotheses of Theorem 4.7 and keep the notation of that result. Then:

(i) $R$ is a Noetherian k-algebra.

(ii) C ${ }^{\prime}{ }_{Y}-\bmod$ is equivalent, both to $\overline{g r}-R$ and to $R-\overline{g r}$. Under this equivalence, the sheaf $\mathscr{M}$ over $Y$ corresponds to the right module $M=\bigoplus_{i \geqq 0} H^{0}(Y, \mathscr{U}$ $\left.\otimes \mathscr{L}_{n}\right)$.

(iii) If the automorphism $\sigma$ associated to $R$ has finite order, then $R$ is a finite module over its centre.

Proof. By Theorem 4.7, $B / R$ is finite dimensional and so it suffices to prove the corollary for $B$. In this case, parts (i) and (ii) are proved in $[\mathrm{AV}]$, while part (iii) follows from [ST, Corollary 2.3].

As will be shown in Sect. 5, a version of Corollary 4.8(ii) holds for any connected graded Noetherian domain $R$ with $G K \operatorname{dim}(R)=2$. However, if $R$ is not generated in degree one, then part (iii) need not hold. For example, let $K=\mathbb{C}(u)$ with automorphism $\sigma$ defined by $\sigma(u)=-u$. Set

$$
R=\mathbb{C}\left\langle z, w z^{2}, w^{3} z^{2}\right\rangle \subset K\left[z, z^{-1} ; \sigma\right] \quad \text { where } w=(1+u) .
$$

Then, $R$ is a finitely graded, Noetherian, PI domain, with $G K \operatorname{dim}(R)=2$, but $R$ is not a finite module over its centre. Since ungraded analogues of this example are well-known, the details of this example are left to the reader. 
As a second application of Theorem 4.7, we complete our discussion of rings for which the associated automorphism $\sigma$ has finite order. In this result we do not assume that $R_{1} \neq 0$.

Theorem 4.9. Let $R$ be a finitely graded domain with $G K \operatorname{dim}(R)=2$ and graded quotient ring $K\left[z, z^{-1} ; \sigma\right]$, where $K=k(X)$ and $z \in Q(R)_{1}$. Assume that $|\sigma|=d<\infty$. The following are equivalent:

(i) $R$ is Noetherian,

(ii) The Veronese ring $R^{(d)}$ is a finitely generated commutative ring,

(iii) Some Veronese ring $R^{(/)}$is generated in degree one. Assume, that $R_{1} \neq 0$ and adopt the notation of (2.1). Then the above conditions are equivalent to

(iv) The Veronese sequence $\left\{D_{n}^{(d)}\right\}$ is a divisor sequence for the automorphism id $=\sigma^{d}$.

A detailed analysis of how part (iv) of this theorem can fail is given by Propositions 2.6 and 2.8. An example of a ring for which the equivalent conditions of Theorem 4.9 do not hold is given by Example 2.5 in characteristic two.

Proof. Lemma 4.10 below shows that the Veronese subring $R^{(d)}$ of a graded domain is Noetherian if and only if $R$ is. In our situation, $R^{(d)}$ is a subring of $K\left[z^{d}, z^{-d} ; \sigma^{d}\right]$, which is commutative because $\sigma^{d}=i d$. Thus $R^{(d)}$ is commutative, and it is Noetherian if and only if it is finitely generated. If so, then some Veronese ring $R^{\left(d^{\prime}\right)}$ is generated in degree one [Mu, Lemma, p. 282]. The equivalence of (i)-(iii) follows from Theorem 4.7.

Suppose that $R_{1} \neq 0$. Then (ii) $\Rightarrow$ (iv) follows from Lemma 2.3. Conversely, suppose that $\left\{D_{m d}\right\}$ is a $\sigma^{d}$-divisor sequence. Then Proposition 2.8 shows that, for some multiple $k$ of $d, D_{m k}=m Z$ for large $m$. Because of Proposition 3.3, we may apply Theorem 4.1. We conclude that $R^{(k)}$ has finite index in some twisted coordinate ring $B(Y, \mathscr{L}, 1)$. So $R^{(k)}$ is Noetherian and finitely generated. Assertion (i) follows from Lemma 4.10.

\section{Lemma 4.10.}

(i) A right Noetherian, graded k-algebra $S$ is a finitely generated $S_{0}$-algebra.

(ii) If $S$ is a right Noetherian graded algebra, then every Veronese subring $S^{(/)}$is right Noetherian.

(iii) A graded domain $S$ such that some Veronese $S^{(f)}$ is right Noetherian is a finite right $S^{(t)}$-module, and hence is right Noetherian.

Proof: The first assertion follows from [Bo, III, Sect. 2, Proposition 1]. The second is $[\mathrm{AZ}$, Proposition 5.10(1)]. To prove (iii), we denote by $\bar{r}$ the residue class of $r$, modulo $\ell$, and we set $S_{\bar{r}}=\bigoplus_{n} S_{n /+r}$, so that $S=\bigoplus_{r=0}^{-1} S_{\bar{r}}$. If $S_{\bar{r}} \neq 0$, then $S_{-\bar{r}} \neq 0$ as well. Left multiplication by a nonzero element $t \in S_{-\bar{r}}$ defines an injective map $S_{\bar{r}} \rightarrow S_{\overline{0}}$ which is right $S_{\overline{0}}$-linear. Now $S_{\overline{0}}$ is just the 
Veronese ring $S^{(f)}$, with a different grading. So $S$ is isomorphic to a finite sum of regraded right ideals of $S^{(\ell)}$.

\section{The case of an automorphism of infinite order}

It remains to consider the structure of rings $R$ which are not generated in degree one and for which the associated automorphism $\sigma$ has infinite order. The basic aim is to derive results that are the analogues of Theorem 4.1 and those of $[A V]$ for these rings. If the gap divisor is zero, then some Veronese ring $R^{(d)}$ is generated in degree one, and so $R$ has the basic properties of a twisted homogeneous coordinate ring. The more interesting case is when the gap divisor is non-zero. In this case, $R$ is similar, at least asymptotically, to the ring $A$ of Example 2.5 in characteristic zero; as with that example, $R$ looks like an idealizer inside a twisted homogeneous coordinate ring (see Theorem 5.9), yet $\vec{R}_{n}=H^{0}\left(Y, \mathscr{R}_{n}\right.$ ) for $n \gg 0$ (Proposition $5.4(\mathrm{i})$ ), and the quotient category $R-\overline{\mathrm{gr}}=(R-\mathrm{gr}) / \tau(R)$ is equivalent to $\mathcal{C}_{Y}-\bmod$ (Theorem 5.11).

To avoid problems at the fixed points, we often assume that $R$ satisfies (2.15). Recall that, if $R$ is any ring satisfying (2.1), with $|\sigma|=\infty$, then Lemma 2.7 and Corollary 2.13 imply that $(2.15)$ will always hold for some Veronese ring $R^{(f)}$. We do not assume $R$ finitely generated, because there is no a priori reason why the Veronese ring of a finitely generated $k$-algebra should itself be finitely generated. However, finite generation does follow from our hypotheses (see Theorem 5.6).

We are interested in the asymptotic structure of right $R$-modules $M$, so we will work in $\overline{\text { gr}}-R$, i.e., with the tails $M_{\geqq n}$ of graded modules for sufficiently large $n$, ignoring irregularities which may occur in low degree. Thus, we usually work with indices $i \geqq n_{1}$, in the notation of (3.7) and we keep the notation developed there. In particular, for $i \geqq n_{1}, \mathscr{R}_{i}$ is an invertible sheaf on the stable image $Y$ of $X$ and the relations (3.8) hold. As at the end of Sect. 3, we will work mainly with fractional ideals.

If $M$ is a nonzero right ideal of $R=\bigoplus \bar{R}_{i} z^{i}$ then, following previous notation, we write $M_{i}=\bar{M}_{i} z^{i}$, and $\mathscr{M}_{i}=\bar{M}_{i} \mathcal{O}_{Y}$. Thus, $\mathscr{M}_{l} \subseteq \mathscr{R}_{i}=\bar{R}_{i} \mathcal{O}_{Y}$, and

$$
\mathscr{M}_{i} \otimes \mathscr{R}_{j}^{\sigma^{t}} \subseteq \mathscr{M}_{i+j} \text { for } j \gg 0
$$

For $i \geqq n_{1}$, set $\mathscr{J}_{i}=\mathscr{J}_{i}(M)=\mathscr{M}_{i} \otimes \mathscr{R}_{i}^{\vee}$. Thus $\mathscr{J}_{i} \subseteq \mathscr{R}_{i} \otimes \mathscr{R}_{i}^{\vee}=\mathscr{O}_{Y}$; that is, $\mathscr{F}_{i}$ is an ideal in $\mathscr{O}_{Y}$.

Lemma 5.2. Let $M$ be a nonzero right ideal of $R$, and let $\bar{M}_{i}, \mathscr{M}_{1}$ be as above. Then:

(i) $\mathscr{M}_{i} \otimes \mathscr{B}_{j}^{\sigma^{\prime}}=\mathscr{M}_{i+j}$ for $i, j \gg 0$ where, by definition, $\mathscr{B}_{j}=\mathscr{R}_{j}(\Omega)=$ $\mathscr{R}_{j} \otimes \mathscr{O}_{Y}(\Omega)$.

(ii) $\mathscr{J}_{i}$ is independent of $i \gg 0$.

Proof. Let $Z_{n}$ be the closed subscheme of $Y$ whose ideal sheaf is $\mathscr{J}_{n}$. We first show that the support of $Z_{n}$ is contained in a finite set $S$ independent of $n \gg 0$. 
Using Lemma 3.8(ii), and taking $n=n_{1}$, one obtains

$$
\begin{aligned}
\mathscr{I}_{n}\left(-\sigma^{-n} \Omega\right) & =\mathscr{H}_{n} \otimes \mathscr{R}_{n}\left(\sigma^{-n} \Omega\right)^{\vee} \\
& =\mathscr{M}_{n} \otimes \mathscr{R}_{j}^{\sigma^{n}} \otimes \mathscr{R}_{n+j}^{\vee} \subseteq \mathscr{M}_{n+1} \otimes \mathscr{R}_{n+j}^{\vee}=\mathscr{J}_{n+j} .
\end{aligned}
$$

Since this is independent of $j$, we can take $S=\operatorname{Supp}\left(Z_{n_{1}}\right) \cup \operatorname{Supp}\left(\sigma^{-n_{1}} \Omega\right)$.

Next we show that $\mathscr{M}_{i} \otimes \mathscr{B}_{j}^{\sigma^{\prime}} \subseteq \mathscr{M}_{i+j}$ for large $i, j$. By (5.1), this is true at all points $q$ of $Y$ at which $\mathscr{B} \sigma_{j}^{\sigma^{l}}=\mathscr{R}_{j}^{\sigma^{\prime}}$; that is, for all $q \notin \operatorname{Supp}\left(\sigma^{-1} \Omega\right)$. By Lemma 3.8(iii), it is also true at those points at which $\mathscr{M}_{i}=\mathscr{R}_{i}$ and $\mathscr{M}_{i+j}=\mathscr{R}_{i+j}$. This includes all points $q \notin S$. For large $i, S \cap \operatorname{Supp}\left(\sigma^{-i} \Omega\right)=\emptyset$ and so $\mathscr{M}_{i} \otimes \mathscr{B}_{j}^{\sigma^{\prime}} \subseteq \mathscr{M}_{i+j}$.

Now set $e_{l}=$ length $\left(\mathcal{O}_{Z_{l}}\right)$, and $e=\liminf \left(e_{i}\right)$. By the last paragraph and Lemma 3.8(iii),

$$
\mathscr{I}_{i}=\mathscr{M}_{i} \otimes \mathscr{B}_{j}^{\sigma^{d}} \otimes \mathscr{R}_{i+1}^{\vee} \subseteq \mathscr{M}_{i+j} \otimes \mathscr{R}_{i+j}^{\vee}=\mathscr{I}_{i+j}, \text { for all } i, j \gg 0 .
$$

Thus $e_{i} \geqq e_{i+1}$, and if we choose $i$ so that $e_{i}=e$, then $e_{i+j}=e$ too. This forces the inclusion of (5.2.1) to be an equality. Both assertions of the lemma follow.

Lemma 5.3. Keep the above notation and assume that $n$ is large enough that $\Omega \cap \sigma^{-n} \Omega=0$. Then:

$$
\mathscr{M}_{i} \otimes \mathscr{R}_{j n}^{\sigma^{t}}+\mathscr{M}_{i+n} \otimes \mathscr{R}_{(j-1) n}^{\sigma^{++n}}=\mathscr{M}_{i+j n} \text { for all } i, j \gg 0 .
$$

Proof. The inclusion $\subseteq$ follows from (5.1). Lemma 5.2(i) shows that $\mathscr{M}_{i} \otimes$ $\mathscr{R}_{j n}^{\sigma^{l}}=\mathscr{M}_{i+j n}$ at all points $q \in Y$ at which $\mathscr{R}_{j n}^{\sigma^{l}}=\mathscr{B}_{j n}^{\sigma^{i}}$; that is, at all points $q \notin \operatorname{Supp}\left(\sigma^{-i} \Omega\right)$. Similarly, $\mathscr{M}_{i+n} \otimes \mathscr{R}_{(j-1) n}^{\sigma^{+n}}=\mathscr{M}_{i+j n}$ holds at all points at which $\mathscr{R}_{(j-1) n}^{\sigma^{i+n}}=\mathscr{B}_{(j-1) n}^{\sigma^{i+n}}$, and these are the points $q \notin \operatorname{Supp}\left(\sigma^{-(i+n)} \Omega\right)$. By the choice of $n$, these two sets cover $Y$.

We are now ready to prove the desired analogue of Theorem 4.1.

Proposition 5.4. Under Hypothesis 2.15, let $M$ be a nonzero right ideal of $R$.

(i) For large $i, \bar{M}_{i}=H^{0}\left(Y, \mathscr{M}_{i}\right)$. In particular, $\bar{R}_{i}=H^{0}\left(Y, \mathscr{R}_{i}\right)$ for large $i$.

(ii) For $n \gg 0$, we have $M_{i+j n}=M_{i} R_{j n}+M_{i+n} R_{(j-1) n}$ if $i, j \gg 0$.

Proof. We use the notation of Proposition 2.11. Let $n \geqq n_{1}$ be an integer. After possibly increasing $n$, we may choose $s \in \mathbb{N}$ such that, on each infinite orbit of $\sigma$, and with $a, b$ defined as in (2.11), we have $a+b \leqq s \leqq n-a-b$. We may also assume that $\operatorname{deg}\left(\mathscr{R}_{n}\right)>\operatorname{deg}(\Omega)$. Let $T$ denote the subring of $K\left[z^{n}, z^{-n} ; \sigma^{n}\right]$ generated by $\bar{R}_{n} z^{n}$, write $T=\bigoplus_{j \geqq 0} \bar{T}_{j} z^{n j}$, and let $\mathscr{T}_{j}=\bar{T}_{j} \Theta_{Y}$. Thus $\mathscr{T}_{1}=\mathscr{R}_{n}$, and for $i>1$.

$$
\mathscr{T}_{i}=\mathscr{R}_{n} \otimes \mathscr{R}_{n}^{\sigma^{n}} \otimes \cdots \otimes \mathscr{R}_{n}^{\sigma^{(i-1) n}} .
$$

By the choice of $n, \mathscr{R}_{n}$ and $\mathscr{T}_{i}$ are invertible sheaves on $Y$. 
Lemma 5.5. Under the above hypotheses, $\mathscr{T}_{i}+\mathscr{T}_{i-1}^{\sigma^{\prime}}=\mathscr{R}_{\text {in }}$.

Proof. By Lemma 3.8, $\mathscr{R}_{(i-1) n}^{\sigma^{\prime}} \subseteq \mathscr{R}_{i n}$ and $\mathscr{T}_{i} \subseteq \mathscr{R}_{i n}$ for all $i$. Hence $\mathscr{T}_{1}+$ $\mathscr{T}_{1-1}^{\sigma^{*}} \subseteq \mathscr{R}_{i n}$. To prove the lemma, we may verify equality locally at each point $q \in Y$. Locally at the fixed points, $\mathscr{R}_{i n}=\mathscr{T}_{t}$ by Lemma $3.8(\mathrm{i})$, so equality holds there. Thus it suffices to verify the equality on an infinite orbit of $\sigma$ and hence, by Lemma 3.4, it suffices to check the corresponding fact about the divisor sequence. The divisor corresponding to $\mathscr{T}_{i}$ is $U_{i}:=D_{n}+$ $\sigma^{-n} D_{n}+\cdots+\sigma^{-(i-1) n} D_{n}$, and the relation which must be verified is $U_{i} \cup \sigma^{-s}$ $U_{l-1}=D_{l n}$.

Clearly, $U_{i} \cup \sigma^{-s} U_{t-1} \leqq D_{i n}$. We prove the opposite inequality by induction on $i$. Now, $U_{i}=D_{n}+\sigma^{-n} U_{i-1}$. Thus,

$$
\begin{aligned}
U_{i} \cup \sigma^{-s} U_{l-1} & =\left[D_{n}+\sigma^{-n} U_{t-1}\right] \cup\left[\sigma^{-s} D_{n}+\sigma^{-s-n} U_{t-2}\right] \\
& \geqq F:=\sigma^{-n} D_{(i-1) n} \cup D_{n} \cup \sigma^{-s} D_{n} .
\end{aligned}
$$

The choice of $s$ and (2.12.2) ensure that $\left.\sigma^{-s} D_{n}\right|_{\natural} \geqq\left.\sigma^{-n} \Omega\right|_{\&}$ for each infinite orbit $\mathscr{I}$ of $\sigma$. Thus, by (2.12.1), $F=D_{i n}$, as required.

Proof of Proposition $\leqq .4$, continued. Theorem 4.1 applies to the ring $T$. It shows that

$$
\bar{T}_{i}=H^{0}\left(Y, \mathscr{T}_{i}\right) \text { for } i \gg 0
$$

By Lemma 5.5, there is an exact sequence

$$
0 \rightarrow \mathscr{H} \rightarrow \mathscr{T}_{i} \oplus \mathscr{T}_{i-1}^{\sigma^{\prime}} \rightarrow \mathscr{R}_{i n} \rightarrow 0
$$

The kernel $\mathscr{H}$ is an invertible sheaf because the three other terms in the sequence are invertible, and we have

$$
\begin{aligned}
\operatorname{deg}(\mathscr{H}) & =\operatorname{deg}\left(\mathscr{T}_{i}\right)+\operatorname{deg}\left(\mathscr{T}_{i-1}\right)-\operatorname{deg}\left(\mathscr{R}_{\text {in }}\right) \\
& =(2 i-1) \operatorname{deg}\left(\mathscr{R}_{n}\right)-\operatorname{deg}\left(\mathscr{R}_{i n}\right) \\
& =(i-1) \operatorname{deg}\left(\mathscr{R}_{n}\right)-(i-1) \operatorname{deg}(\Omega) \geqq i-1 .
\end{aligned}
$$

If $i$ is sufficiently large, $H^{1}(Y, \mathscr{H})=0$. Thus, by taking global sections of (5.4.2) and using (5.4.1) one finds that $\bar{T}_{i}+\bar{T}_{i-1}^{\sigma^{s}}=H^{0}\left(Y, \mathscr{R}_{i n}\right)$. On the other hand, by construction, $\bar{T}_{i}+\bar{T}_{i-1}^{\sigma^{\prime}} \subseteq \bar{R}_{i n}$. We have thus shown that $\bar{R}_{m}=$ $H^{0}\left(Y, \mathscr{R}_{i n}\right)$ for $i, n \gg 0$.

We now use a similar argument on $M$. Fix $i_{0} \gg 0$ and $i_{0} \leqq i \leqq i_{0}+n$. Since $\bar{M}_{i}$ generates $\mathscr{M}_{i}$, we have exact sequences

$$
0 \rightarrow \mathscr{K}_{j} \rightarrow \bar{M}_{i} \otimes_{k} \mathscr{R}_{j n}^{\sigma^{l}} \rightarrow \mathscr{M}_{i} \otimes \mathscr{R}_{j n}^{\sigma^{\prime}} \rightarrow 0
$$

for all $j \gg 0$. Lemma 3.8 (iii) shows that $\mathscr{K}_{j+\ell}=\mathscr{K}_{j} \otimes \mathscr{B}_{i n}^{\sigma^{i+j n}}$ for all $k \gg 0$. Since $\operatorname{deg}\left(\mathscr{B}_{/ n}\right)$ is large when $\ell$ is large, this implies that $H^{1}\left(Y, \mathscr{K}_{j}\right)=0$ if $j \gg 0$. Moreover, by the last paragraph, $H^{0}\left(Y, \bar{M}_{i} \otimes_{k} \mathscr{n}_{j n}^{\sigma^{l}}\right)=\bar{M}_{i} \otimes_{k} \bar{R}_{j n}$. Thus, 
taking global sections of the last displayed equation shows that the natural map $\bar{M}_{i} \otimes_{k} \bar{R}_{j n} \rightarrow H^{0}\left(Y, \mathscr{H}_{i} \otimes \mathfrak{R}_{m}^{\sigma^{l}}\right)$ is surjective for $j \gg 0$.

By Lemma 5.3, there is also an exact sequence

$$
0 \rightarrow \mathscr{H}_{j} \rightarrow\left(\mathscr{H}_{1} \otimes \mathscr{K}^{\sigma^{l}}\right) \oplus\left(\mathscr{M}_{i+n} \otimes \mathscr{K}_{(j-1) n}^{\sigma^{++n}}\right) \rightarrow \mathscr{H}_{i+j n} \rightarrow 0
$$

Once again, $\mathscr{H}_{j+t}=\mathscr{H}_{j} \otimes \mathscr{B}_{n}^{\mathrm{s}^{1+}}$; hence $H^{1}\left(Y, \mathscr{H}_{j}\right)=0$ if $j \gg 0$. Combined with the last paragraph, this shows that, for $j \gg 0$, the natural map

$$
\phi: \bar{M}_{l} \otimes \bar{R}_{j n}+\bar{M}_{i+n} \otimes \bar{R}_{(j-1)_{n}} \rightarrow H^{0}\left(Y, \mathscr{M}_{1+m}\right)
$$

is surjective. Finally, since $\operatorname{Im}(\phi) \subseteq \bar{M}_{t+j n}$, this implies that $\bar{M}_{i+j n}=H^{0}(Y$, $\mathscr{U}_{1+j n}$ ) and that $\bar{M}_{1+m}=\bar{M}_{i} \bar{R}_{j n}^{\sigma^{t}}+\bar{M}_{i+n} \bar{R}_{(j-1) n}^{\sigma^{\prime+n}}$, for all $i_{0} \leqq i \leqq i_{0}+n$ and all $j \gg 0$. This completes the proof of both parts of Proposition 5.4.

Theorem 5.6. Let $R$ be a finitely graded domain with $G K \operatorname{dim}(R)=2$ and quotient ring $K\left[w, w^{-1} ; \tau\right]$ and assume that $|\tau|=\infty$. We do not assume that $R_{1} \neq 0$. Then, every Veronese ring $R^{(d)}$ is Noetherian, and hence finitely generated. In particular, $R$ is Noetherian.

Proof. According to Lemma 4.10, it suffices to prove that some Veronese subring is Noetherian. Choose $d$ so that $R_{d} \neq 0$. Proposition 2.21 shows that the divisor sequence associated to $R^{(d)}$ is a $\sigma^{d}$-divisor sequence. Passing to a multiple of $d$, if required, Hypothesis 2.15 will hold for $R^{(d)}$. Thus we are reduced to the case that $(2.15)$ holds for $R$. Then the fact that a right ideal $M$ of $R$ is finitely generated follows from Proposition 5.4(ii). Hence $R$ is right Noetherian. The fact that $R$ is left Noetherian follows from the next lemma.

Lemma 5.7. If $R$ satisfies Hypothesis 2.15 , then so does its opposite ring $R^{\circ p}$.

Proof. We identify the sets $R$ and $R^{\circ p}$, and denote multiplication in $R^{\circ p}$ by $\circ$. Define $\bar{R}_{i}^{\mathrm{op}}$ by $R_{i}=\bar{R}_{i}^{\mathrm{op}} \circ z^{i}=z^{i} \bar{R}_{i}^{\mathrm{op}}$. Then $\bar{R}^{\mathrm{op}}=\bar{R}_{i}^{\sigma^{-l}}$. Thus if $D_{i}^{\mathrm{op}}$ denotes the $\sigma$-divisor sequence associated to $R^{\circ p}$, then $D_{i}^{\circ p}=\sigma^{i} D_{i}$. Of course, the automorphism $\sigma$ is replaced by its inverse in the opposite ring. Hypotheses 2.14 and 2.15 carry over.

As was shown in Sect. 4 , if $R$ is generated in degree one then, up to a finite dimensional vector space, $R$ is a twisted homogeneous coordinate ring. If $R$ satisfies Hypothesis 2.15 but the gap divisor of $R$ is non-zero, then this is not true (see Proposition 6.6, for example). Nevertheless, as we show in Theorem 5.9, $R$ is still closely related to a twisted coordinate ring and this, again, can be used to give a detailed description of the module structure of $R$.

Lemma 5.8. Let $R$ be a ring that satisfies Hypothesis 2.15. Take $\mathscr{B}_{i}$ and $n_{1}$ as in (3.7). Then:

(i) $\mathscr{L}:=\mathscr{B}_{i+1} \otimes\left(\mathscr{B}_{i}^{\sigma}\right)^{\vee}$ is a sheaf that is independent of $i \gg 0$. 
(ii) If $\mathscr{L}_{n}=\mathscr{L} \otimes \mathscr{L}^{\sigma} \otimes \cdots \otimes \mathscr{L}^{\sigma^{t-1}}$, then $\mathscr{L}_{i}=\mathscr{B}_{i}$ for $i \gg 0$. Thus, $\mathscr{L}$ is an invertible sheaf that is both $\sigma$-ample and $\sigma^{-1}$-ample.

(iii) $\mathscr{R}_{i} \otimes \mathscr{L}_{j}^{\sigma^{l}}=\mathscr{R}_{i+j}$ for all $i \geqq n_{1}$ and $j \geqq 1$.

Proof. (i) This follows from the computation

$$
\mathscr{B}_{i+j+1} \otimes\left(\mathscr{B}_{i+j}^{\sigma}\right)^{\vee}=\left(\mathscr{B}_{i+1} \otimes \mathscr{B}_{j}^{\sigma^{i+1}}\right) \otimes\left(\left(\mathscr{B}_{l}^{\sigma}\right)^{\vee} \otimes\left(\mathscr{B}_{j}^{\sigma^{\alpha+1}}\right)^{\vee}\right)=\mathscr{B}_{i+1}^{\sigma} \otimes\left(\mathscr{B}_{i}^{\sigma}\right)^{\vee},
$$

which is valid for $i, j \geqq n_{i}$.

(ii) For $i, j \gg 0$, we have

$$
\begin{aligned}
\mathscr{L}_{i}=\left(\mathscr{B}_{i+j} \otimes\left(\mathscr{B}_{i+j-1}^{\sigma}\right)^{\vee}\right) \otimes\left(\mathscr{B}_{i+j-1} \otimes\left(\mathscr{B}_{i+j-2}^{\sigma}\right)^{\vee}\right)^{\sigma} \\
\otimes \cdots \otimes\left(\mathscr{B}_{j+1} \otimes\left(\mathscr{B}_{j}^{\sigma}\right)^{\vee}\right)^{\sigma^{-1}} \\
=\mathscr{B}_{i+j} \otimes\left(\mathscr{B}_{j}^{\sigma^{l}}\right)^{\vee}=\mathscr{B}_{i} .
\end{aligned}
$$

Since $\mathscr{B}_{i}$ is ample for large $i$, this implies that $\mathscr{L}$ is also ample. Therefore, by [AV, Corollary 1.6], $\mathscr{L}$ is both $\sigma$-ample and $\sigma^{-1}$-ample.

(iii) Fix $i \geqq n_{1}$ and $j \gg 1$. Then, by Lemma 3.8(iii, v), we have

$$
\begin{aligned}
\mathscr{R}_{i} \otimes \mathscr{L}^{\sigma^{\prime}} & =\mathscr{R}_{i} \otimes \mathscr{B}_{j+1}^{\sigma^{l}} \otimes\left(\mathscr{B}_{j}^{\sigma^{++1}}\right)^{\vee}=\mathscr{R}_{i+j+1} \otimes\left(\mathscr{B}_{j}^{\sigma^{+1}}\right)^{\vee} \\
& =\mathscr{R}_{i+1} \otimes \mathscr{B}_{j}^{\sigma^{\prime+1}} \otimes\left(\mathscr{B}_{j}^{\sigma^{\prime+1}}\right)^{\vee}=\mathscr{R}_{i+1} .
\end{aligned}
$$

The result follows by induction.

Theorem 5.9. Let $R$ be a ring that satisfies Hypothesis 2.15 and let $B=$ $B(Y, \mathscr{L}, \sigma)$, for the sheaf $\mathscr{L}$ defined by Lemma 5.8. Then:

(i) $B$ contains $R$ and is a Noetherian right $R$-module.

(ii) As left $R$-module, $B / R$ is torsion.

(iii) $B$ is a Noetherian left $R$-module if and only if the gap divisor $\Omega$ is zero.

Proof. We first show that $R \subseteq B$, for which it suffices to prove that $\mathscr{R}_{i} \subseteq \mathscr{L}_{i}$, for all $i \geqq 1$. Suppose that $\mathscr{R}_{j} \nsubseteq \mathscr{L}_{j}$. Since $\mathscr{L}$ is invertible, Lemma 5.8(ii) implies that

$$
\mathscr{B}_{i} \otimes \mathscr{R}_{j}^{\sigma^{l}}=\mathscr{L}_{i} \otimes \mathscr{R}_{j}^{\sigma^{l}} \nsubseteq \mathscr{L}_{i} \otimes \mathscr{L}_{j}^{\sigma^{l}}=\mathscr{B}_{i+j},
$$

for all $j \gg 0$. This contradicts Lemma 3.8(i) and hence proves that $R \subseteq B$.

By Proposition 5.4 and Lemma 5.8, there exists $m$ such that

$$
\bar{R}_{i} \bar{B}_{j}^{\sigma^{d}} \subseteq H^{0}\left(Y, \mathscr{R}_{i} \otimes \mathscr{L}_{j}^{a^{i}}\right) \subseteq H^{0}\left(Y, \mathscr{R}_{i+j}\right)=\bar{R}_{i+j},
$$

for $i, j \geqq m$. Thus, $z^{m} B_{\geqq m} \subset R$ and, since $R$ is Noetherian, both $B \geqq m$ and $B$ are finitely generated right $R$-modules.

The same formula shows that $\left(R_{\geqq m}\right)^{2} B \subseteq R_{\geqq m} B_{\geqq m} \subset R$. Since $R$ is left Noetherian, this implies that $B / R$ is a torsion left $R$-module. 
If the gap divisor is zero, then $\mathscr{R}_{i}=\mathscr{B} \mathscr{B}_{i}$, and Proposition 5.4 implies that $R_{i}=B_{1}$ for large $i$. Thus $R$ has finite codimension in $B$. Conversely, if the gap divisor $\Omega$ is not zero, then $\mathscr{R}_{1} \neq \mathscr{B}_{1}$, for large $i$, and the Riemann-Roch Theorem implies that $R_{l} \neq B_{l}$. Therefore $\operatorname{dim}_{k}(B / R)=\infty$. Since $B / R$ is a torsion left $R$ module, it cannot be finitely generated.

Lemma 5.2(ii) allows us to associate a closed subscheme of $Y$ to a right ideal $M$ of $R$; set $\mathscr{J}(M)=\mathscr{I}_{i}(M)$ for large $i$, and define $Z(M)$ to be the closed subscheme whose ideal sheaf is $\mathscr{J}(M)$. In fact, this is part of the more general equivalence of categories. Let $\mathscr{F} \in \mathscr{O}_{Y}$-mod, the category of coherent sheaves of $C_{Y}$-modules. Set

$$
\bar{M}_{i}(\tilde{\mathscr{F}})=H^{0}(Y, \bar{F} \otimes \mathscr{R} i) \text {, and } M=M(\mathscr{F})=\bigoplus_{i \geqq 0} \bar{M}_{i} z^{i} \text {. }
$$

By Lemma 3.8(i), $M$ is a right $R$-module.

Theorem 5.11. Let $R$ be a graded ring which satisfies Hypothesis 2.15 Then:

(i) The map $\mathscr{F} \mapsto \bigoplus_{1 \geq 0} H^{0}\left(Y, \mathscr{F} \otimes \mathscr{R}_{l}\right)$ defines an equivalence of categories between $\mathcal{O}_{Y}$-mod and $\overline{\mathrm{gr}}-\mathrm{R}$.

(ii) Under this correspondence, the $\sigma$-invariant ideals of $\mathscr{C}_{Y}$ correspond to the classes of two sided graded ideals of $R$.

Proof. (i) When $\mathscr{J}$ is an ideal of $\mathcal{O}_{Y}$, it follows easily from Lemma 5.2(ii) and Proposition 5.4 that the map of part (i) is the inverse of the functor $M \rightarrow \mathscr{J}(M)$. In order to prove this for other coherent sheaves, one can either generalize $[\mathrm{AV}]$ to cover the present situation or use [SZ]. We will use the latter method since it is quicker, although this requires us to work with left modules. Thus, we consider the map

$$
\alpha: \mathscr{\mathscr { F }} \mapsto M_{R}(\mathscr{F})=\bigoplus_{i \geqq m} H^{0}\left(Y, \mathscr{R}_{i} \otimes \mathscr{\mathscr { H }}^{\sigma^{\prime}}\right) z^{l} .
$$

By the analogue of Lemma 3.8(i), $\alpha(\mathscr{F})$ is a left $R$-module and we wish to prove that $\alpha$ induces an equivalence of categories $\mathcal{O}_{Y}$-mod $\rightarrow R$-gr. Part (i) of the theorem will then follow by applying Lemma 5.7. Note that, since we are interested in the image of $\mathscr{F}$ in $R$ - $\overline{\mathrm{gr}}$, the choice of the integer $m$ in (5.11.1) is irrelevant and it will be convenient to take $m \gg 0$. Let $B=B(Y, \mathscr{L}, \sigma)$, as before.

By $\left[\mathrm{AV}\right.$, Theorem 3.12], the map $\beta: \mathscr{F} \mapsto M_{B}(\widetilde{\mathscr{F}})=\bigoplus_{i \geqq m} H^{0}\left(Y, \mathscr{L}_{i} \otimes\right.$ $\left.\mathscr{F} \sigma^{i}\right) z^{i}$ gives an equivalence of categories $\mathcal{O}_{Y}$-mod $\rightarrow B$-gr. Next, let $I=$ $\bigoplus_{l \geq m} H^{0}\left(Y, \mathscr{R}_{i}\right) z^{i}$. By Lemma 5.8(iii), $I$ is a right ideal of $B$ and, by Proposition 5.4. $I=R_{\geqq m}$. By [SZ, Lemma 2.6 and Proposition 2.7], the map $\gamma: M_{B}(\mathscr{F}) \rightarrow I M_{B}(\mathscr{F})$ provides an equivalence of categories $B$ - $\overline{\mathrm{gr}} \rightarrow R$ - $\overline{\mathrm{gr}}$. Thus, it remains to prove that $\alpha=\gamma \circ \beta$.

Note that $I M_{B}(\mathscr{F})_{r}$ is the image in $M_{B}(\mathscr{F})$, under the multiplication map, of the space

$$
\sum_{\substack{i+1=r \\ i, I \geq m}} H^{0}\left(Y, \mathscr{R}_{i}\right) \otimes H^{0}\left(Y, \mathscr{L}_{j}^{\sigma^{l}} \otimes \mathscr{F}^{\sigma^{++1}}\right) z^{r}
$$


Since $m \gg 0$, Lemma 5.8(iii) and Proposition 5.4 imply that this is contained in $M_{R}(\tilde{\mathscr{F}})_{r}=H^{0}\left(Y, \mathscr{R}_{r} \otimes \mathscr{F}^{r}\right) z^{r}$. Moreover, by Lemma $4.2($ iii), the map

$$
H^{0}\left(Y, \mathscr{R}_{l}\right) \otimes H^{0}\left(Y, \mathscr{L}_{j}^{\sigma^{l}} \otimes \mathscr{F}^{\sigma^{l+1}}\right) z^{r} \rightarrow M_{R}(\mathscr{F})_{i+j}
$$

is surjective for all $i \gg 0$. Thus, there exists $t \gg 0$ such that $\gamma \circ \beta(\mathscr{F})_{\geqq t}=$ $M_{R}(\mathscr{F})_{\geqq l}=\alpha(\mathscr{F})_{\geqq l}$, as required.

(ii) Let $M$ be a nonzero, two-sided ideal. Then, with the usual notation, $\bar{R}_{i} \bar{M}_{j}^{\sigma^{\prime}} \subseteq \bar{M}_{1+j}$, and this implies that $\mathscr{R}_{i} \otimes \mathscr{M}_{j}^{\sigma^{\prime}} \subseteq \mathscr{M}_{1+j}$ for large $i, j$. Tensoring with $\mathscr{R}_{i+j}^{\vee}$ and using Lemma 3.8(ii), we obtain the relation $\mathscr{f}_{j}^{\prime}\left(-\sigma^{-l} \Omega\right) \subseteq$ $\mathscr{I}_{i+j}$, for $i, j \gg 0$. By Lemma 5.2(ii), this implies that $\mathscr{J}^{\sigma^{t}}\left(-\sigma^{-i} \Omega\right) \subseteq \mathscr{J}$. Thus $\mathscr{J}^{\sigma^{l}} \subseteq \mathscr{J}$ is true at all points $q \notin \operatorname{Supp}\left(\sigma^{-l} \Omega\right)$. It is also true at points at which $\mathscr{J}=\mathscr{O}_{Y}$; that is, at points not in the support of $Z$. Since $\operatorname{Supp}(Z) \cap$ $\operatorname{Supp}\left(\sigma^{-i} \Omega\right)=\emptyset$ for $i \gg 0$, this implies that $\mathscr{J}^{\sigma^{\prime}} \subseteq \mathscr{J}$ for such $i$. Thus $\mathscr{I}$ is $\sigma$-invariant.

Conversely, let $\mathscr{J}$ be a $\sigma$-invariant ideal, define $\mathscr{M}_{j}=\mathscr{I} \otimes \mathscr{R}_{j}$ and set $\bar{M}_{j}=\bar{M}_{j}(\mathscr{J})$ and $M=M(\mathscr{I})$. Clearly, $M_{\geqq n}$ is a right ideal of $R$, for any $n \gg 0$. Then,

$$
\mathscr{R}_{i} \otimes \mathscr{M}_{j}^{\sigma^{\prime}}=\mathscr{R}_{i} \otimes \mathscr{J} \otimes \mathscr{R}_{j}^{\sigma^{l}}=\mathscr{J} \otimes \mathscr{R}_{i} \otimes \mathscr{R}_{j}^{\sigma^{l}} \subseteq \mathscr{M}_{i+j} \quad \text { for all } i \gg 0 .
$$

Taking into account Proposition 5.4, this shows that $\bar{R}_{i} \bar{M}_{j}^{\sigma^{\prime}} \subseteq \bar{M}_{i+j}$, for all $i, j \gg 0$ and hence that $M_{\geqq n}$ is a left ideal for some $n \gg 0$.

Remark $\leqq .12$. (i) The following special case of part (i) of Theorem 5.11 will be used several times: To $p \in Y$, or rather its associated skyscraper sheaf, part (i) of the theorem associates a point module; that is an $R$-module $M=M(p)$ with $\operatorname{dim} M_{i}=1$ for all $i \geqq 0$. The analogous comment applies to Corollary 4.8 .

(ii) Lemma 5.8 allows one to rewrite the equivalence of part (i) of the theorem as $\mathscr{F} \mapsto \bigoplus_{i \geqq 0} H^{0}\left(Y, \widetilde{\mathscr{F}} \otimes \mathscr{L}_{i}\right)$, where $\widetilde{\mathscr{F}}=\mathscr{F} \otimes \mathscr{R}_{n_{1}} \otimes \mathscr{L}_{n_{1}}^{-1}$.

\section{Applications to the structure of graded domains of dimension two}

In this section we use the earlier results of this paper to describe the structure of an arbitrary, Noetherian, connected graded domain $R$ of Gelfand-Kirillov dimension two. The natural way to do this is to relate $R$ to a suitable Veronese $\operatorname{ring} R^{(d)}$, and then to apply Theorems 4.9 and 5.11. But since the module categories of these two rings are not equivalent, some care is required.

We begin with a discussion of Veronese rings in general. Let $A$ be a graded

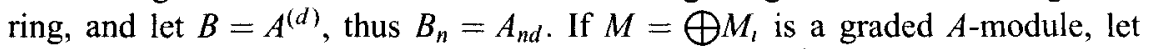
$M^{(d)}$ be the graded $B$-module defined by $M^{(d)}=\bigoplus_{i} M_{i}^{(d)}$, for $M_{i}^{(d)}=M_{d i}$. Note that these definitions have the technical disadvantage that one must regrade $B$ 
and $M^{(d)}$ if one wishes to regard $B$ as a graded subring of $A$ and $M^{(d)}$ as a graded $B$-submodule of $M$.

Assume that $A$ is right Noetherian. Then, by Lemma $4.10, B$ is also right Noetherian. Let gr- $A$ denote the category of Noetherian, graded right $A$ modules, with homomorphisms being graded homomorphisms of degree zero. There is a pair of adjoint functors

$$
V: \operatorname{gr}-A \rightarrow \operatorname{gr}-B, \quad T: \operatorname{gr}-B \rightarrow \operatorname{gr}-A,
$$

defined by $V(M)=M^{(d)}$, and $T(N)=N \otimes_{B} A$, where $T(N)$ is given the natural graded structure defined by $T(N)_{t}=\sum_{1} N_{1} \otimes A_{t-d}$ ( (see [Ve] for the proof when $A$ is generated by $A_{1}$ and $A_{0}$ ). Thus

$$
\operatorname{Hom}_{B}(N, V(M)) \cong \operatorname{Hom}_{A}(T(N), M) .
$$

The functor $V$ is exact, and $T$ is right exact, full and faithful. Let $\mathscr{C}$ denote the full subcategory of gr- $A$ consisting of graded right modules such that $V(M)=$ 0 . Then, $\mathscr{C}$ is a dense subcategory of gr- $A$, and gr- $B$ is equivalent to the quotient category $\mathrm{gr}-A / \mathscr{C}$. We have $V T \cong i d$, the kernel and cokernel of the canonical map $T V(M) \rightarrow M$ are in $\mathscr{C}$, and $T V(A) \cong A$.

Recall that a nonzero graded $A$-module $M$ is called $i$ - critical if $G K \mathrm{dim}$ $(M)=i$ and $G K \operatorname{dim}(\bar{M})<i$ for every quotient $\bar{M}$ of $M$.

Proposition 6.1. Let $A$ be a right Noetherian graded ring, which is a finite left and right module over its Veronese subring $B=A^{(d)}$.

(i) For any finitely generated, graded $B$-module $N, G K \operatorname{dim}(N)=G K$ $\operatorname{dim}(T(N))$. If $M$ is a finitely generated, graded $A$-module which can be generated by elements whose degrees are in $d \mathbb{Z}$, then $G K \operatorname{dim}(M)=G K$ $\operatorname{dim}(V(M))$.

(ii) If $M \in \mathrm{gr}-A$ is $i$-critical, then $V(M)$ is either the zero module, or else it is $i$-critical.

(iii) For any $N \in \operatorname{gr}-B$, there is a maximal submodule $C \subset T(N)$ with $C \in \mathscr{C}$, and $\tilde{N}=T(N) / C$ has the property that $V(\tilde{N}) \cong N$. If $N$ is i-critical, then $\tilde{N}$ is i-critical.

(iv) The function $\sim$ sending $N \leadsto \tilde{N}$ is a bijection from the set of $i$-critical $B$-modules to the set of i-critical A-modules which are generated in degrees $d \mathbb{Z}$. Its inverse function is $V$.

(v) Let $N \in \operatorname{gr}-B$ be an i-critical module with (prime) annihilator $Q$, and suppose that $G K \operatorname{dim}(B / Q)=i$. Let $P \subset A$ be the annihilator of the i-critical $A$-module $\tilde{N}$ defined in (iii). Then $G K \operatorname{dim}(A / P)=i$.

Proof. (i) For $N \in \operatorname{gr}-B$, we have $G K \operatorname{dim}(N) \leqq G K \operatorname{dim}(T(N))$ because $N \subset T(N)$. The other inequality follows from [KL, Proposition 5.6].

If $M \in \operatorname{gr}-A$ is generated in degrees $d \mathbb{Z}$, then the canonical map $T V(M)$ $\rightarrow M$ is surjective. Hence $G K \operatorname{dim}(M) \geqq G K \operatorname{dim}(V(M))=G K \operatorname{dim}(T V(M))$ $\geqq G K \operatorname{dim}(M)$, which shows that $G K \operatorname{dim}(M)=G K \operatorname{dim}(V(M))$.

(ii) Let $M \in \mathrm{gr}-A$ be an $i$-critical module such that $V(M) \neq 0$, and let $\psi: B \rightarrow V(M)$ be a nonzero map with cokernel $N$. We must show that 
GK $\operatorname{dim}(N)<i$. Let $\phi$ denote the adjoint map $T(B)=A \rightarrow M$. This map is not zero, and it factors through $T(\psi)$, and provides a row exact commutative diagram

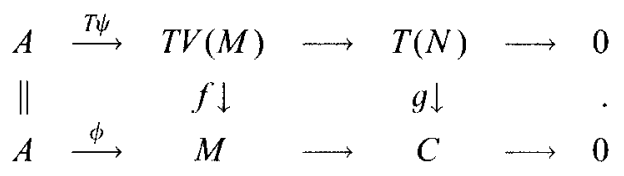

Since $M$ is critical, $G K \operatorname{dim}(C)<i$. From the diagrarm, one deduces that the map $\operatorname{ker} f \rightarrow \operatorname{ker} g$ is surjective, and hence that $\operatorname{ker} g \in \mathscr{C}$. Applying $V$ to the map $g$ yields an exact sequence $0 \rightarrow V($ ker $g) \rightarrow N \rightarrow V(C)$. Since $V(\operatorname{ker} g)=0$, this shows that $G K \operatorname{dim}(N)<i$, as required.

(iii) It is clear that the maximal submodule $C \subset T(N)$ with $C \in \mathscr{C}$ exists, and then $V(C)=0$, hence $V(\tilde{N}) \simeq V T(N) \simeq N$. Suppose that $N$ is $i$-critical. If $S=\tilde{N} / I$ is a proper factor of $\tilde{N}$, then $V(S) \cong V(\tilde{N}) / V(I)=N / V(I)$, and $V(I) \neq 0$, by the definition of $\mathscr{C}$. Hence $G K \operatorname{dim}(V(S))<i$. Since $T(N)$ is generated by $N$, it is generated in degrees $d \mathbb{Z}$, and so is $S$. By (i), $G K \operatorname{dim}(S)<i$, and this shows that $\tilde{N}$ is $i$-critical.

(iv) Items (ii) and (iii) show that the maps $\sim$ and $V$ carry $i$-critical modules to $i$-critical ones. Since $\tilde{N}=T(N) / C$ with $C \in \mathscr{C}, V(\tilde{N})=V T(N)=N$. For the composition in the other order, let $M$ be an $i$-critical $A$-module generated in degrees $d \mathbb{Z}$, and set $N=V(M)$. Let $\tilde{N}=T(N) / C$ as in (iii), and let $\bar{C}$ be the image of $C$ via the surjective map $T(N) \rightarrow M$. Then, since $N=$ $V(M)=V(M / \bar{C})$ and $M$ are $i$-critical, part (i) implies that $G K \operatorname{dim}(M / \bar{C})=$ $i=G K \operatorname{dim}(M)$. Since $M$ is critical, $\bar{C}=0$. So the surjective map $T(N) \rightarrow M$ factors through $\tilde{N}$. Since $\tilde{N}$ is $i$-critical and $M$ has GK-dimension $i$, the map $\tilde{N} \rightarrow M$ is bijective. This shows that $\sim$ and $V$ are inverse functions.

(v) The module $N$ is a homomorphic image of a sum of shifts of $B / Q$, and so $\tilde{N}$ is a homomorphic image of a sum of shifts of $M:=T(B / Q)=A / Q A$. Regarding $M$ as an (ungraded) ( $B, A)$-bimodule, we have $\ell$-ann $(M)=Q$. Let $I=r$-ann $(M)$. Then by [KL, Lemma 5.3], $i=G K \operatorname{dim}(B / Q)=G K \operatorname{dim}(M)=$ $G K \operatorname{dim}(A / I)$. Since $I \subset P=r-\operatorname{ann}(\tilde{N})$, it follows that $G K \operatorname{dim}(A / P) \leqq i$. Since $G K(\tilde{N})=i$, the opposite inequality also holds.

We now return to rings of dimension two. Since we are interested in rings that need not contain elements of degree one, the following conventions will be in force for the rest of the section:

Notation 6.2. $A$ is a finitely graded, Noetherian domain, with $G K \operatorname{dim}(A)=2$ and graded quotient $\operatorname{ring} Q(A) \cong K\left[t, t^{-1} ; \tau\right]$, where $K=k(X)$ is a function field in one variable. By regrading $A$, if necessary, we will assume that $t \in$ $Q(A)_{1}$. By Theorem 4.9 , respectively Theorem 5.6 , we may choose an integer $d$ such that, if $R=A^{(d)}$, then either $R$ is a commutative ring generated in degree one (and $|\tau|<\infty$ ) or $R$ is a ring that satisfies (2.15) (in which case $|\tau|=\infty)$. We define $\sigma=\tau^{d}$ and let $Y$ be the curve associated to $R$ in Sect. 3 . 
Recall that, if $|\tau|=\infty$ or if $A$ is generated in degree one, then the Noetherian hypothesis in (6.2) is automatic, by Theorems 5.6 and 4.9. If $k$ is algebraically closed, then Theorem 1.1 implies that the hypothesis that $Q(A) \cong K\left[t, t^{-1} ; \tau\right]$ is also automatic.

Corollary 6.3. Let $A$ and $R$ satisfy the hypotheses of (6.2).

(i) Let $M$ be a 1-critical graded right $A$-module. Then for $n \gg 0, \operatorname{dim} M_{n}$ is a periodic function of $n$ whose values are taken in the set $\{0,1\}$.

(ii) The 1-critical A-modules that are generated in degrees $d \mathbb{Z}$ are in one-to-one correspondence with the points of $Y$.

Proof. (i) Because of (6.1)(ii) and the possibility of shifting degrees, it suffices to prove this for the Veronese ring $R=A^{(d)}$. The result now follows from Remark 5.12 combined with the equivalence of categories given by Corollary 4.8(ii), respectively Theorem 5.11(i).

(ii) By Proposition 6.1(iv), the 1-critical $A$-modules generated in degrees $d \mathbb{Z}$ are in one-to-one correspondence with those over $R$ and hence, by Corollary 4.8(ii) and Theorem 5.11(i), again, are in one-to-one correspondence with the points of $Y$.

If $A$ is the ring of Example 2.5 in characteristic zero, then, for each $n>0$, there exists a cyclic, 1-critical $A$-module with Hilbert series $\frac{1}{(1-1)}-t^{n}$ ([SZ, Lemma 3.4]). Thus, in part (i) of Corollary 6.3, it can take arbitrarily long before $\operatorname{dim} M_{n}$ settles down. The second part of the corollary raises an intriguing question: does there exist a natural structure on the set of 1-critical $A$-modules generated in degree zero that makes this set into a variety isomorphic to $Y$ ? If $A$ is generated in degree one, then this is true and is the philosophy behind the variety of point modules considered in [ATV]. However, the structure used in that paper does not work for Example 2.5 (see $[\mathrm{SZ}]$ ) and so a more subtle approach is needed in the general case.

If $A$ and $R$ are defined as in (6.2), then Theorem 4.7 and Proposition 5.4 imply that each $R_{n}$ is isomorphic to the vector space of global sections of the appropriate sheaf of $O_{Y}$-modules. This also holds for $A$ :

Proposition 6.4. Let $A=\bigoplus_{n \geqq 0} A_{n}$ and $R=A^{(d)}$ be defined as in (6.2). Then:

(i) There exist coherent sheaves $\mathscr{L}, \mathscr{F}_{0}, \ldots, \mathscr{F}_{d-1}$ over $Y$, with $\mathscr{L}$ invertible, such that, for all $0 \leqq i \leqq d-1$ and $n \gg 0$,

$$
A_{n d+i} \cong H^{0}\left(Y, \mathscr{F}_{i} \otimes \mathscr{L} \otimes \mathscr{L}^{\tau^{d}} \otimes \cdots \otimes \mathscr{L}^{t^{(n-1) d}}\right)
$$

(ii) There exist integers $a_{i}, b_{l} \geqq 0$ such that $\operatorname{dim} A_{n d+i}=a_{i} n-b_{i}$, for all $0 \leqq i<d$ and all $n \gg 0$.

Proof. (i) We return to the notation used in the proof of Lemma 4.10: $A=$ $\bigoplus_{r=0}^{d-1} A_{\bar{r}}$, where $\bar{r}$ is the residue class of $r$ modulo $d$. Regrading suitably, we may regard each $A_{\bar{r}}$ as a graded right $R$-module. Depending on whether $|\sigma|=1$ or $\infty$, let $\mathscr{L}$ be the invertible sheaf defined Theorem 4.7 or Theorem 5.11. Now 
apply Remark 5.12 together with either Corollary 4.8 or Theorem 5.11 to the $R$-modules $A_{\bar{r}}$.

(ii) This follows easily from part (i) and the Riemann-Roch Theorem.

One can obviously give considerably more information about how the ring structure of $A$ affects the sheaves $\mathscr{F}_{i}$ in this result. However, even when $A$ is commutative, the $\mathscr{F}_{i}$ need not be invertible (see the example mentioned before Lemma 3.4).

Recall, for example from Corollary 1.3, that $A$ satisfies a polynomial identity if and only if $|r|<\infty$. The next corollary makes this dichotomy more extreme.

Proposition 6.5. Let $A$ satisfy the hypotheses of (6.2) and assume that $|\tau|=$ $\infty$. Then:

(i) $A$ is primitive.

(ii) If the associated curve $Y$ is elliptic then the only prime ideals of $A$ are 0 and $A_{+}$.

(iii) If $Y$ is not elliptic, it is rational. In this case, $A$ has one or two prime ideals $P_{j}$ with $G K \operatorname{dim}\left(A / P_{j}\right)=1$. Both are graded. If $Q$ is any other non-zero prime ideal of $A$, then $A / Q$ is finite dimensional and $Q \supset P_{j}$ for one of these graded primes $P_{j}$.

Proof. (ii, iii) If $P$ is a non-zero graded prime ideal of $A$, then either $G K$ $\operatorname{dim}(A / P)=1$ or $P=A_{+}$(use [KL, Theorem 2.5]). Let $R=A^{(d)}$ be defined by (6.2) and write $\mathscr{P}(R)$ for the set of graded prime ideals $Q$ of $R$ such that $G K \operatorname{dim}(R / Q)=1$. Recall from the discussion prior to (2.9) that the only finite $\sigma$ orbits in $Y$ are the fixed points. If $Q \in \mathscr{P}(R)$, then Theorem 5.11 applies, and it shows that, ignoring terms of low degree, there is a unique 1-critical $R / Q$-module of GK-dimension 1, namely $N=R / Q$ itself. So setting $f(Q)=P$, where $P$ is the annihilator of $\tilde{N}$ as in Proposition 6.1(v), defines a single valued map $f: \mathscr{P}(R) \rightarrow \mathscr{P}(A)$.

To show that the function $f$ is surjective, let $P \in \mathscr{P}(A)$ and let $M$ be a 1-critical graded right module whose annihilator is $P$. By Proposition 6.1(ii), $V(M)$ is either 1-critical or zero. Shifting $M$ as necessary, we may assume that $V(M) \neq 0$. The right annihilator of $V(M)$ in $A$ is a prime ideal $Q$ which contains $V(P)$. This construction provides a right inverse to $\varepsilon$. By the discussion before (2.9), this implies that $\mathscr{P}(A)=\emptyset$ if $Y$ is elliptic and that $\mathscr{P}(A)$ contains one or two primes if $Y$ is rational. This proves (ii) and (iii) for graded prime ideals.

Let $Q$ be an ungraded, prime ideal of $A$. If $A / Q$ is finite dimensional, then the remarks after [SSz, Lemma 4.1] imply that $Q \supset P$, for some graded prime ideal $P \in \mathscr{P}(A)$. If $G K \operatorname{dim}(A / Q)=1$, then [SW] implies that $A / Q$ is a finite module over its affine centre and so $Q=\bigcap M$, where $M$ runs throughout the maximal ideals of $A$ containing $Q$. But each such $M$ contains the finite intersection $W=A_{+} \cap \bigcap\left\{P_{i}: P_{i} \in \mathscr{P}(A)\right\}$. Thus, $Q=P_{i}$, for some such $i$.

(i) If $w \in W \backslash\{0\}$ and $M$ is any maximal (left or right) ideal or $R$ containing $1-w$, then parts (ii) and (iii) imply that $R / M$ is a faithful simple $R$-module. Thus, $A$ is primitive. 
A finitely graded ring $S$ is said to satisfy condition $\chi_{1}$ if $\operatorname{dim} \operatorname{Ext}_{S}^{1}\left(S / S_{+}, S\right)$ $<\infty$. This condition is central to the study of $\overline{\mathrm{gr}}-S$ in [AZ]. If $S$ is a Noetherian PI ring, then $\chi_{1}$ is automatic, by [AZ, Theorem 8.13], and so the next result determines precisely when this condition holds for domains of dimension two.

Proposition 6.6. Let $A$ be defined as in (6.2) and assume that $|\tau|=\infty$. Then:

(i) The following conditions are equivalent: (a) some Veronese ring $A^{(n)}$ is generated in degree one, (b) the gap divisor $\Omega$ of $A^{(d)}$ is zero, (c) dim $\mathrm{Ext}_{A}^{1}$ $(k, A)<\infty$.

(ii) There exists $n$ such that the Veronese ring $A^{(n)}$ is generated in degrees one and two.

Proof. (i) Observe that, if $A^{(n)}$ is generated in degree one, then so is $A^{(n d)}$. Similarly, the gap divisor for $A^{(d)}$ is zero if and only if the gap divisor of $A^{(n d)}$ is zero. Moreover, by Lemma 4.10 and the proof of [AZ, Proposition 8.7], the Ext condition holds for $A$ if and only if it holds for $A^{(m d)}$. Thus, it suffices to prove the result for $R=A^{(n d)}$.

If $\Omega=0$, then Lemma 3.8(ii) implies that $\mathscr{R}_{m u}+\mathscr{R}_{i}^{q^{m t}}=\mathscr{R}_{(m+1) i}$ for $i \gg 0$. It follows from Proposition 5.4 that, for large $i, R^{(i)}$ is generated in degree one. The Ext condition for $R^{(i)}$ and hence for $R$ follows from Theorem 4.7 combined with [AZ, Theorem 4.5].

If $\Omega \neq 0$, then (2.16) implies that $D_{i}+\sigma^{i} D_{1} \neq D_{2 l}$, for any $i>0$, and so $R^{(i)}$ cannot be generated in degree one. Also, Theorem 5.9 produces a ring $B$, such that $B / R$ is infinite dimensional but $R_{\geq m} B \subseteq R$. This implies that $\operatorname{dim} \operatorname{Ext}_{R}^{\dagger}(M, R)=\infty$, where $M$ is the finite dimensional right $R$-module $R / R_{\geqq m}$. By induction, $\operatorname{dim} \operatorname{Ext}_{R}^{1}(k, R)=\infty$.

We leave it to the reader to reduce assertion (ii) to Corollary 2.13(ii).

One of the major aims of [SZ] was to produce an example (to wit, Example 2.5 in characteristic zero) of a Noetherian ring $A$ for which $\operatorname{dim} \operatorname{Ext}_{A}^{1}(k, A)=$ $\infty$, since this provides a useful counterpoint to the positive results of [AZ]. Of course, this also follows from Proposition 6.6. We have used that same example many times in this paper, simply because it is an archetypal example of a ring with non-zero gap divisor. We end the paper by noting that the concepts of the last two sections can be used to show the profusion of such rings:

Example 6.7. Pick a projective curve $Y$ with an infinite automorphism $\sigma$. Write $\phi: X \rightarrow Y$ for the normalization of $Y$ and let $U_{X}$ denote the union of the infinite orbits of $\sigma$. Pick a $\sigma$-divisor sequence $\left\{D_{n}\right\}$ supported on $U_{X}$ for which the gap divisor sequence $\Omega$ is non-zero. For example, one can fix an effective divisor $\Omega>0$ supported on $U_{X}$ and define $D_{n}$ inductively by $D_{1}=0$ and $D_{n+1}=D_{n}+\sigma^{n} D_{1}+\sigma^{n} \Omega$ for $n \geqq 1$. Since $D_{n}$ is supported on $U_{X} \cong U_{Y}$, it defines an invertible sheaf $\mathcal{O}_{Y}\left(D_{n}\right)$ over $Y$. Thus, we may consider $R=$ $k+\bigoplus_{n \geqq 1} H^{0}\left(Y, \mathcal{O}\left(D_{n}\right)\right) z^{n}$. Condition (2.15) is trivially satisfied by $R$ and so this ring satisfies all the conclusions of Theorems 5.6, 5.9 and 5.11 and Proposition 6.6 (with $\Omega \neq 0$ ). 


\section{References}

[ATV] M. Artin, J. Tate, M. Van den Bergh: Some algebras associated to automorphisms of curves. In: The Grothendieck Festschrift P. Cartier et al. (eds.), Birkhäuser: Basel, 1990

[AV] M. Artin, M. Van den Bergh: Twisted homogeneous coordinate rings. J. Algebra 133, 249-271 (1990)

[AZ] M. Artin, J.J. Zhang: Noncommutative projective schemes. Adv. in Math. 109, 228287 (1994)

[Be] G.M. Bergman: A note on growth functions of algebras and semigroups, mimeographed notes, University of California, Berkeley, 1978

[Bo] N. Bourbaki: Éléments de Mathématique, Algèbre Commutative. Hermann: Paris 1961

[Co] P.M. Cohn: Algebra II. Wiley: Chichester, 1977

[GW] K.R. Goodearl, R.B. Warfield, Jr.: An Introduction to Noncommutative Noetherian Rings, London Math. Soc. Student Texts No. 16, CUP: Cambridge, 1989

[Ha] R. Hartshorne: Algebraic Geometry. Springer: Berlin/ New York (1977)

[IS] R.S. Irving, L.W. Small: The Goldie conditions for algebras with bounded growth. Bull. London Math. Soc. 15, 596-600 (1983)

[KL] G. Krause, T.H. Lenagan: Growth of Algebras and Gelfand-Kirillov Dimension. Pitman: London, 1985

[LVV] L. Le Bruyn, M. Van den Bergh, F. Van Oystaeyen: Graded orders. Birkhäuser: Boston, 1988

[MR] J.C.Mcconnell, J.C. Robson: Noncommutative Noetherian Rings. Wiley-Interscience: Chichester, 1987

[ML] L. Makar-Limanov: The skew field of fractions of the Weyl algebra contains a free subalgebra. Comm. in Algebra 11, 2003-2006 (1983)

[Mu] D. Mumford: The Red Book on Varieties and Schemes. (Lecture Notes in Math. No. 1358). Springer-Verlag: Berlin/New York, 1988

[NV] C. Năstăsescu, F. Van Oystaeyen: Graded Ring Theory. North Holland: Amsterdam, 1982

[Se] J.-P. Serre: Faisceaux Algébriques Cohérents. Ann. Math. 61, 197-278 (1955)

[SW] L.W. Small, R.B. Warfield: Jr: Prime affine algebras of Gelfand-Kirillov dimension one. J. Algebra 91, 386-389 (1984)

[SSz] S.P. Smith, J.M. Staniszkis: Irreducible representations of the 4-dimensional Sklyanin algebra at points of infinite order. J. Algebra 160, 57-86 (1993)

[ST] S.P. Smith, J. Tate: The center of the three and four dimensional Sklyanin algebras. K-Theory 8, 19-64 (1994)

[St] J.T. Stafford: On the ideals of a Noetherian ring. Trans. Amer. Math. Soc. 289, 381-392 (1985)

[SZ] J.T. Stafford and J.J. Zhang: Examples in noncommutative projective geometry. Math. Proc. Camb. Phil. Soc. 116, 415-433 (1994)

[Ve] A.B. Verevkin: On a noncommutative analogue of the category of coherent sheaves on a projective variety. Amer. Math. Soc. Transl. 151, 41-53 (1992) 